Jens Bemme \& Martin Munke

\title{
Open Citizen Science: Leitbild für kuratorische Praktiken in Wissenschaftlichen Bibliotheken
}

Bibliotheken und Bibliotheksmitarbeiter/-innen bieten Ressourcen: Wissen, Räume, Personal und Beratung, Methoden, Zeit und Orientierung. Für das Handlungsfeld Citizen Science zeigen die Autoren im Folgenden beispielhaft, wie Entscheidungen über solche Ressourcen, ihre Bearbeitung und Benutzung, Verknüpfung und Rekombination sowie die betreffende Kommunikation kuratorische Praktiken beinhalten. Sie zeigen außerdem, dass Verknüpfungen offener Daten im Sinne von Linked Open Data nicht auf digitale Räume beschränkt bleiben. Herausforderungen bestehen für Bibliotheken insbesondere darin, für die Bürgerwissenschaften reale und digitale Räume zu verbinden und diverse $\mathrm{Zu}$ gänge zu ermöglichen, denn Digital Information Literacy - die Fähigkeit mit digitalen Informationen souverän, verantwortlich und zielgerichtet umzugehen - ist für viele Bürger/-innen weitgehend Neuland. Sie unterscheiden sich damit nicht grundsätzlich von Bibliotheksangestellten. Die Aneignungen offener Bibliotheks- und digital offener Arbeitsumgebungen sollen nicht nur möglich sein; Offenheit und Kollaboration sind vielmehr die Voraussetzung und zugleich das Ziel für Forschungsprozesse in der Bürgerwissenschaft. Idealerweise sind Bibliotheksmitarbeiter/-innen deshalb zugleich Bürgerwissenschaftler/-innen, um Wissen für Citizen Science zu vermitteln. Die Autoren argumentieren vor dem Hintergrund ihrer Tätigkeiten in der Sächsischen Landesbibliothek - Staatsund Universitätsbibliothek Dresden (SLUB) und aus der Perspektive der Sächsischen Landeskunde.

\section{Offene Kulturdaten als Gegenstand kuratori- scher Praktiken}

Der Dresdner Ingenieur Andreas Wagner ${ }^{1}$ arbeitet seit September 2019 in seiner Freizeit jeden Sonnabend am Wikisource-Informationsstand in der Sächsischen Landesbibliothek - Staats- und Universitätsbibliothek Dresden (SLUB) an Tran-

1 Rück 2018.

ə Open Access. (C) 2021 Jens Bemme \& Martin Munke, publiziert von De Gruyter. (@)BY Dieses Werk ist lizenziert unter der Creative Commons Attribution 4.0 Lizenz.

https://doi.org/10.1515/9783110673722-013 
skriptionen im deutschsprachigen Portal von Wikisource ${ }^{2}$ - und er berät dort Nutzer/-innen zu dieser ehrenamtlichen Tätigkeit. An seinem Beispiel wird deutlich, wie an offenen Kulturdaten orientierte Citizen-Science-Initiativen von Angeboten einer Bibliothek profitieren - und darüber hinaus von weiteren Ressourcen der SLUB. Zugleich profitieren die Bibliothek als Institution und ihre Mitarbeiter/-innen durch die Kooperation.

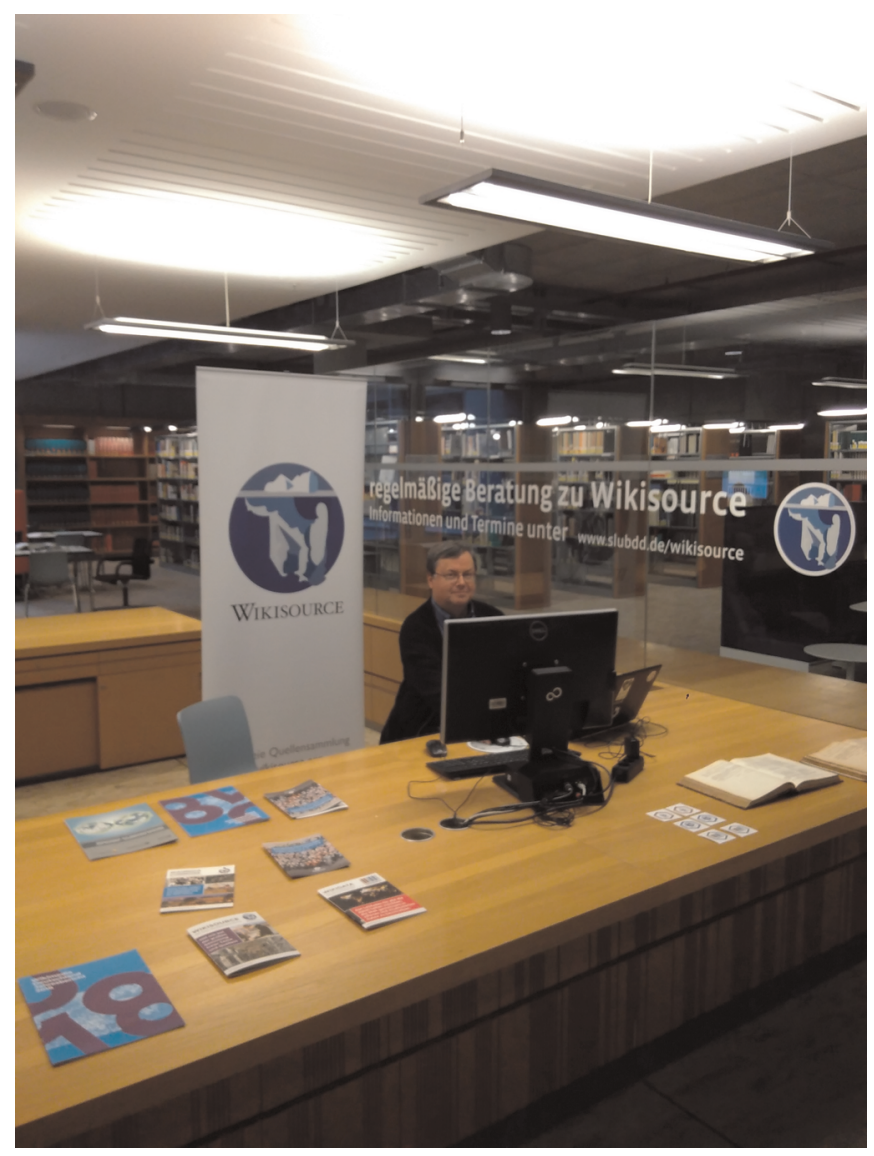

Abb. 1: Andreas Wagner am Wikisource-Beratungsstand in der SLUB Dresden. ${ }^{3}$ Foto: Mr. N, CC BY-SA.

2 https://de.wikisource.org/wiki/Wikisource:Wikisource-Informationsstand_SLUB; https:// www.slub-dresden.de/open-science/citizen-science/wikisource-beratung/ (09.01.2020). 3 https://commons.wikimedia.org/wiki/File:Wikisource-Infostand-Dresden.jpg (31.05.2020). 
Andreas Wagners Engagement wirft Fragen auf, die Bürgerwissenschaften in Bibliotheken betreffen, z. B.: Wie hängen Bibliotheksräume und digitale Arbeitsumgebungen zusammen? Inwieweit kann seine Arbeit mit Wikisource als Blaupause fungieren für Citizen-Science-Kooperationen in und mit Bibliotheken? Was bedeutet Citizen Science im Zusammenhang mit der digitalen Transformation für das bibliothekarische Arbeiten? ${ }^{4}$

Andreas Wagner pflegt Kulturdaten: historische und zeitgenössische Texte darunter viele Saxonica - unter anderem aus den gemeinfreien Katalogen der Jahresausstellungen der Kurfürstlich- bzw. Königlich-Sächsischen Akademie der Künste ${ }^{5}$ sowie Daten und Texte internationaler Kulturabkommen. Er koordiniert und administriert Wikisource in Deutschland mit, und er hält für die deutschsprachige Wikisource-Community Kontakt zum Verein Wikimedia Deutschland e. V. Die SLUB profitiert davon durch Know-how in Bezug auf kollaborative Werkzeuge, durch Projektbeispiele für die Weiterverwendung und Bearbeitung von Objekten aus digitalen Sammlungen, durch Wissenszuwachs für die bibliografische Erschließung digitaler - und insbesondere landeskundlicher - Texte und Bilder mittels Wikidata sowie durch die öffentliche Profilierung in der Open-GLAM-Bewegung bei gleichzeitigem Ausbau der „Vernetzung mit den historisch verwandten Gedächtniseinrichtungen Archiv und Museum“. ${ }^{6}$ Diese Verknüpfungen analoger Bibliotheksarbeit und analoger Bibliotheksräume in den verschiedenen Standorten der SLUB mit - davon erst einmal unabhängigen - digitalen Projekten und Text- und Datenproduktionen in Wikipedia, Wikisource, Wikidata sowie darüber hinaus in Stadt- und Regiowikis und in anderen Gedächtnisinstitutionen ist das Ergebnis mehrjähriger Netzwerkarbeit, Kommunikation und Anbahnung ${ }^{7}$ : prozess- und ressourcenorientiert, ergebnisoffen, interessengeleitet und kollaborativ über die institutionellen Grenzen der SLUB Dresden hinweg. Seinen Ausdruck gefunden hat dieser Ansatz auch im Leitbild des 2019 veröffentlichten Strategiepapiers der SLUB: „Wissen teilen Menschen verbinden". 8

Bürgerwissenschaftlicher Umgang mit offenen Kulturdaten und die zur Nachnutzung und zur weiteren Bearbeitung offenen Präsentation der Ergebnis-

4 Vgl. dazu auch Audunson et al. 2019.

5 https://de.wikisource.org/wiki/Kataloge_der_Jahresausstellungen_der_Kurfurstl./Königl. _Sächsischen_Akademie_der_Künste (09.01.2020).

6 Das Akronym GLAM steht für die Kulturinstitutionen Galleries, Libraries, Archives, Museums. Für die Möglichkeiten, die die Digitalisierung für eine vertiefte Zusammenarbeit dieser Institutionen bietet, vgl. Marcum 2014. Das Zitat bei T. Bürger 2015, hier: S. 69.

7 Munke \& Bemme 2019, bes. S. 191-194.

8 Bonte \& Muschalek (Hrsg.) 2019. 
se, hier als Open Citizen Science ${ }^{9}$ bezeichnet, verändert das Verhältnis zwischen Bibliotheksnutzer/-innen, Bibliothekspersonal sowie Dritten - Akteuren, die erst aufgrund der Kooperationsbeziehung von Bürgerwissenschaftler/-innen und Bibliotheksmitarbeiter/-innen in offene Forschungsprozesse involviert werden. Schließlich geht mit den aktuellen gesellschaftlichen Prozessen im Kontext der Digitalisierung, „geht mit der Transformation in eine Wissensgesellschaft und der technologischen Entwicklung ein neues Kompetenzportfolio für Bürgerinnen einher" ${ }^{\star 10}$, das gerade von (Wissenschaftlichen wie Öffentlichen) Bibliotheken unterstützt werden kann.

Die Unterscheidung zwischen den genannten Gruppen und ihren Rollen ist dabei nicht immer trennscharf möglich, wenn z. B. an Citizen Science interessierte Bibliotheksmitarbeiter/-innen zugleich bzw. alternierend auch als Bürgerwissenschaftler/-innen denken und handeln. Für die Betrachtung kuratorischer Rollen, Entscheidungen und Praktiken in Forschungsprozessen in Bibliotheken ist folgende Beobachtung grundlegend: Openness ${ }^{11}$ als Haltungs- und Handlungsprinzip in Kooperationen prägt die betreffenden Projekte, deren Forschungsprozesse, die Forschungsergebnisse, die Selbstwirksamkeitserfahrungen der Beteiligten und den individuellen Wissensgewinn.

Möglicherweise ist der Begriff der „Programmierschnittstelle“ (oder: API ${ }^{12}$ ) auch für das Verständnis professioneller und ehrenamtlicher Forschungskooperationen in Bibliotheken verwendbar und hilfreich - über etwaige Übersetzungsund Vermittlungsleistungen zwischen verschiedenen Fachkulturen und Gesellschaftsbereichen hinaus. ${ }^{13}$ Der Geschichtsmarkt Dresden ${ }^{14}$, das TextLab der SLUB $^{15}$, Wikisource-Sprechstunden und Kultur-Hackathons sind Formate, in denen Menschen und Themen in „analogen“ Räumen - also in der umbauten Infrastruktur der Bibliothek - und in digitalen Arbeitsumgebungen zusammenkommen. Schnittstellen zwischen technischen Systemen funktionieren auch dort als Analogie für Verbindungen zwischen Menschen, Räumen und digitalen

9 Bemme \& Munke 2019; Vohland \& Göbel 2017.

10 Tiepmar, Dobeleit \& Mittelbach 2019, hier: S. 95.

$11 \mathrm{Zu}$ „Offenheit“ als Handlungsprinzip für den Bibliotheksbereich vgl. Lohmeier \& Mittelbach 2014; eine allgemeine Einführung bietet Wikimedia Deutschland e. V./Open Knowledge Foundation Deutschland e. V.: ABC der Offenheit, Berlin 2019, URL: https://commons.wikimedia. org/wiki/File:ABC_der_Offenheit_-_Broschüre_(2019).pdf (09.01.2020).

12 Vgl. Art. Programmierschnittstelle, in: Wikipedia. Die freie Enzyklopädie, https://de.wikipedia.org/wiki/Programmierschnittstelle (03.02.2019).

13 In begrifflicher Anlehnung an Nassehi 2016.

14 https://www.geschichtsmarkt-dresden.de (09.01.2020).

15 „Das SLUB TextLab ist der gemeinsame Schreibraum von SLUB, Schreibzentrum und Graduiertenakademie der TU Dresden: eine offene Werkstatt für sämtliche Arbeiten am Text. Ein Makerspace der Worte ...“. URL: https://www.slub-dresden.de/service/textlab/ (09.01.2020). 
Arbeitsumgebungen: Menschen und Bibliotheken, die beide sammeln und forschen und mit diesen Ressourcen anhand von offenen Kulturdaten digitale und reale Räume oder Kommunikation gestalten, um bestehendes Wissen auszutauschen, und neues Wissen schaffen. ${ }^{16}$

Beeinflusst wird die Wirkung dieser kuratorischen Praktiken in Bibliotheken außerdem durch den Faktor Zeit: Programmiersprints im Rahmen von Kulturhackathons verdeutlichen zum Beispiel den Zusammenhang zwischen Zeit (im Sinne von z. B. Dauer und Zeitpunkten) und Wirkung kuratorischer Entscheidungen in Citizen-Science-Projekten. Gewünschte Wirkungen neu entwickelter - in der Regel pilothafter - Software- und Hardwarelösungen mit Daten ausgewählter Kulturgüter bzw. ihrer Metadaten treten in Hackathons deutlich $\mathrm{zu}$ Tage, insbesondere durch gezielt verkürzte Entwicklungszeiträume und -zyklen, z. B. bei Coding da Vinci. ${ }^{17}$ Große Innovationsgeschwindigkeit und ein hoher Innovationsgrad, steigende Sichtbarkeit, Reichweite und Nutzung von Projektergebnissen in GLAM-Institutionen sind beispielsweise solche angestrebten Wirkungen und Ziele.

Im Rahmen von Coding da Vinci wählen Kultureinrichtungen aus, welche Themen, Werke und Daten als Gegenstände für Projekte angeboten werden. Nötig ist häufig eine weitere Aufbereitung dieser Daten, was einen entsprechenden Aufwand mit sich bringt. Bürger/-innen (Hacker/-innen, Designer/-innen u. a.) wählen erst im nächsten Schritt aus den angebotenen Datensätzen. Nicht jeder angebotene Datenbestand weckt Interesse, wird ausgewählt und verwendet. ${ }^{18}$ Darüber hinaus existieren unabhängige Projektportale wie Wikisource und Wikidata, in denen selbstständig digitalisierte Werke oder selbst erhobene Forschungsdaten verwendet werden. Institutionelle OpenGLAM-Strategien, mit denen beide Ansätze i.S. v. Linked Open Data ${ }^{19}$ - also mit persistenten Identifiern versehene, maschinenlesbare, frei verfüg- und nutzbare Daten - verknüpft werden können, bieten zugleich einen hohen Grad an Freiheit für die Aktiven (die hackenden bzw. forschenden Bürger/-innen) wie auch eine jeweils höhere Nutzungswahrscheinlichkeit für die von Kultureinrichtungen angebotenen Datenbestände. Wie nun die Konzepte Citizen Science und Kuratieren in diesem Kontext verstanden werden können, wollen wir im Folgenden versuchen aufzuschlüsseln.

16 Den Möglichkeiten und Herausforderungen bei der Verbindung physischer und virtueller Angebote von Bibliotheken v. a. mit Blick auf Informationsrecherche und -zugang haben sich unter dem Begriff der Blended Library zuletzt einige Studien gewidmet; vgl. Reiterer 2016.

17 Zwischen Auftaktveranstaltung und Ergebnispräsentation liegen durchschnittlich sechs Wochen. Vgl. zur Hackathon-Reihe Fischer 2019; Bartholmei 2014; Bartholmei 2019.

18 Kluttig 2018, S. 23-24.

19 Vgl. für den Bibliothekskontext Pohl \& Danowski 2014, S. 392-409; Neubert 2014. 


\section{Begriffe}

\subsection{Citizen Science in Wissenschaftlichen Bibliotheken}

Noch ist Citizen Science nicht nur für Wissenschaftliche Bibliotheken in Deutschland im Detail ein unbestimmter Begriff. Prototypisch lassen sich mit Peter Finke zwei Ausprägungen unterscheiden:

„eine, die Citizen Science als eine nichtselbständige Form von Wissenschaft auffasst, bei der die Beiträge der Laien letztlich einer Auswertung und Kontrolle durch die Experten bedürfen (meist auch bereits einer Planung durch diese), und eine andere, die sie als eine selbständige, solcher Kontrolle nicht bedürftige Form[,] breit in der Gesellschaft verankerter Wissensbeschaffung versteht. “20

Jenseits dieser Gegenüberstellung, zwischen der viele Abstufungen existieren, erscheint es für Wissenschaftliche Bibliotheken sinnvoll, einem möglichst breiten Ansatz zu folgen. So kann es gelingen, viele Akteure und Akteurinnen anzusprechen - je nach deren eigenen Interessen an einer Beteiligung an bestehenden Projekten oder der Verfolgung eigener Forschungsinteressen. Ein aktueller Beitrag in einer österreichischen Fachzeitschrift definiert entsprechend wie folgt: „Citizen science is a developing method for enhancing the scientific endeavour increasing scientific literacy, supporting education, and better addressing societal needs through scientific evidence. ${ }^{\text {“21 }}$ Die genannten Pole werden dabei nicht aufgegriffen, stattdessen verschiedene Aspekte von Wissenschaftlichkeit betont. Ähnlich sieht auch Peter Finke Citizen Science an anderer Stelle als ein „Modell einer ausschließlich auf Sachinteressen und Fähigkeiten gegründe-

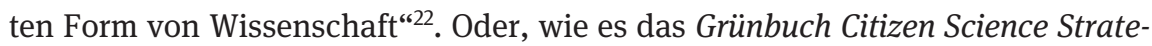
gie 2020 für Deutschland von der Online-Plattform Bürger schaffen Wissen formuliert:

Citizen Science beschreibt die Beteiligung von Personen an wissenschaftlichen Prozessen, die nicht in diesem Wissenschaftsbereich institutionell angebunden sind. [...] Wichtig ist [...] die Einhaltung wissenschaftlicher Standards, wozu vor allem Transparenz im Hinblick auf die Methode der Datenerhebung und die öffentliche Diskussion der Ergebnisse gehören. ${ }^{23}$

20 Finke 2014, S. 42.

21 Ignat, Cavalier \& Nickerson 2019, hier: S. 329.

22 Finke (Hrsg.) 2015, S. 18.

23 Bonn, Richter \& Vohland 2016, S. 13. 
Bei allen Definitions- und Abgrenzungsfragen steht fest: Bürgerwissenschaft findet statt - auch ohne, dass Bibliotheken explizit etwas dafür tun. Bürgerinnen und Bürger forschen und finden als Nutzer/-innen in Bibliotheken dafür Medien, Werkzeuge und Unterstützung. Konzeptionelles strategisches Handeln in Bibliotheken für diese Nutzergruppen ist noch selten. Welche spezifischen Bewertungskriterien werden Citizen-Science-Services in Bibliotheken zugrunde gelegt? ${ }^{24}$ Da sich Bibliotheks- und Sammlungsprofile unterscheiden, dürften sich auch Citizen-Science-Strategien dementsprechend voneinander unterscheiden. Bibliotheken im Gebiet der Biodiversitätsforschung werden naheliegenderweise anders mit Citizen-Science-Projekten kooperieren als Bibliotheken mit wachsenden digitalisierten Sammlungen landeskundlicher, nationaler und internationaler Quellen z. B. in der Kunstgeschichte. Neben den explizit als Citizen Science benannten Aktivitäten ist auch die Forschung aus den deutschen Bibliotheken heraus noch sehr überschaubar - obwohl das Engagement zumindest für Felder wie die Heimat- oder die Familienforschung, die in diesem Kontext gesehen werden können, schon eine lange Tradition aufweist. ${ }^{25}$ Bisherige Studien wie die grundlegenden Arbeiten von Eva Bunge haben hauptsächlich internationale Beispiele für den Vergleich gewählt. ${ }^{26}$ Für einen Teilaspekt wie das Crowdsourcing ist die Literatur auch zu Beispielen aus dem DACH-Raum bedeutend umfangreicher. ${ }^{27}$ Allerdings meint (Open) Citizen Science für uns viel mehr als den hier oft verfolgten Ansatz, mithilfe der Crowd „eigene Datenbestände suchbar und online verfügbar zu machen“ ${ }^{28}$ und damit eigene Wissens- und Ressourcenlücken auszugleichen, mithin die „Auslagerung von Aufgaben durch Unternehmen oder Institutionen an eine nicht näher definierte Masse an Menschen“²9.

Zugleich ist die Rolle, die Bibliotheken für die Bürgerforschung spielen oder spielen könnten, selbst für die professionellen Akteure der Bürgerwissenschaften offenbar noch nicht deutlich genug formuliert und adressiert, als dass Bibliotheken als an Bürgerforschungsprozessen beteiligte Akteure bewusst wahrgenommen werden. ${ }^{30}$ Dies folgt einerseits aus Unkenntnis klassisch bibliothekarischer Aufgaben, Arbeitsweisen und Werkzeuge, z. B. der Formal- und Sacherschließung, der Bedeutung von Metadaten für die Katalogisierung und

24 Munke \& Bemme 2019, S. 183-185.

25 Für die Aktivitäten der SLUB sind zuletzt einige Darstellungen erschienen. Vgl. neben Munke \& Bemme 2019 v. a. Munke 2019a und Munke 2018.

26 Vgl. für die wissenschaftlichen Bibliotheken Bunge 2017, für die öffentlichen Bibliotheken Bunge 2019.

27 Vgl. v. a. die Arbeiten von Graf und Georgy, zuletzt z. B. Graf 2016; Georgy 2019.

28 Georgy 2019, S. 96.

29 Georgy 2018, hier: S. 189.

30 Bemme \& Munke 2019a. 
deren Wirkung für die Auffindbarkeit und die Sichtbarkeit von Wissen. Open Citizen Science eröffnet die Chance durch offene Werkzeuge, die nun für klassische Bibliotheksaufgaben ergänzend zur Verfügung stehen, Nutzer/-innen zu sensibilisieren und zu befähigen, beispielsweise Metadaten als Produkte der eigenen Forschung selbst, schneller und genauer zu erzeugen, Daten zu beschreiben, zu pflegen und bewusster von ihnen zu profitieren.

Offene Methoden und Werkzeuge i.S.v. Open Citizen Science können also helfen, das öffentliche Verständnis kuratorischer sowie an Daten und an Metadaten orientierter Arbeitsweisen in Bibliotheken zu steigern, den Linked-OpenData-Ansatz vieler GLAM-Institutionen zu popularisieren und, allgemein gesprochen, Nutzerteilhabe zu erhöhen. ${ }^{31}$ Selbstständiges Forschen, Datenerheben und bei der Pflege von Forschungsdaten zu helfen, sind Praktiken kreativen und kuratorischen Handelns, das nicht auf das Bibliotheksteam beschränkt bleibt. Der Entwicklung und Programmierung von Abfragen mit dem Wikidata Query Service $^{32}$ und systematischer Skripte für die Datenpflege mit dem Tool QuickStatements ${ }^{33}$ ebenda wohnt in hohem Maße ein selbstständiges Momentum inne - über die zur Bedienung nötigen grundlegenden Programmierkenntnisse hinaus.

\subsection{Kuratieren in Wissenschaftlichen Bibliotheken}

Noch schwieriger wird es begrifflich, wenn von Kuratieren im Kontext von Wissenschaftlichen Bibliotheken die Rede ist - wovon nicht zuletzt der Sammelband zeugt. Im Kontext der digitalen Transformation hat dabei zuletzt das Konzept der Digital Curation an Bedeutung gewonnen. Es bezeichnet nach einer grundlegenden Definition ,actions needed to maintain digital research data and other digital materials over their entire life-cycle and over time for current and future generations of users“334, also Fragen von Langzeitverfügbarkeit, Metadatenstandards, Lizenzmodellen u. ä. Damit erscheint es zunächst als ein technologiegetriebener Ansatz, den Herausforderungen des digitalen Zeitalters zu begegnen. ${ }^{35}$ Es dürfte unbestritten sein, dass angesichts wachsender Datenmengen

31 Zum Ansatz, die Geschichte von Bibliothek als beständiger Erweiterung von Offenheit und Teilhabe zu schreiben, vgl. knapp Bürger 2015, bes. S. 64-67.

32 https://query.wikidata.org (09.01.2020).

33 https://tools.wmflabs.org/wikidata-todo/quick_statements.php (09.01.2020).

34 Beagrie 2006, hier: S. 4.

35 Vgl. Kuwalik 2016. 
und Informationszusammenhänge ${ }^{36}$ sowohl aus quantitativer wie aus qualitativer Perspektive im Sinne von Data Curation nur ein technologiegestütztes Vorgehen helfen kann, klassische bibliothekarische Aufgaben wie Erschließung, Dokumentation und Kontextualisierung zu bewältigen. $\mathrm{Zu}$ den entsprechenden Anwendungen und Verfahren liegen auch mit Bezug auf den Kuratierungsbegriff Untersuchungen vor. ${ }^{37}$

Wir verfolgen demgegenüber eher einen akteurs- und raumzentrierten Ansatz, der natürlich nicht losgelöst von den technologischen Grundlagen ist. Dafür gehen wir von den Überlegungen von Hans Ulrich Obrist aus, die sich zunächst auf das Kuratieren zeitgenössischer Kunst beziehen, die aber auch für den Bibliotheksbereich Anknüpfungspunkte bieten. Obrist sieht für den Kurator bzw. die Kuratorin vier Funktionen: 1. das Bewahren von Werken als Artefakte, „die gemeinsam die Geschichte eines Landes [oder einer Region, einer Stadt etc.; Anm. d. Verf.] erzählen“, 2. die Auswahl neuer Werke für eine Sammlung, 3. die wissenschaftliche Erforschung dieser Werke und 4. ihre Präsentation in Form von Ausstellungen. ${ }^{38}$ Während im zeitgenössischen Gebrauch des Wortes Kuratieren dieses eher auf den vierten Aspekt konzentriert wird ${ }^{39}$, gerät mit Obrists Ansatz ein klassisches Paradigma der Bibliothekswelt wieder in den Blick: das Konzept der Sammlung.

Mit Thomas Stäcker lässt sich die bibliothekarische Sammlung grob auf ein „geordnetes und strukturiertes Ganzes“40 herunterbrechen. Die Prinzipien Ordnung und Strukturierung gelten für einen traditionellen wie für einen aktualisierten Sammlungsbegriff, der den Medienwandel berücksichtigt und digitale Objekte - textueller sowie zunehmend nicht-textlicher Art ${ }^{41}$ - inkludiert. Vorgenommen werden sie in der „vereinheitlichenden formalen Beschreibung“ und der ,inhaltlichen (Sach-)Erschließung “42. Beide Elemente gilt es nun, in maschinenlesbarer Form und in standardisierten Formaten vorzunehmen und vorzuhalten; mit Stäcker: „Sammeln bedeutet in diesem Sinn Transformieren und Homogenisieren nach etablierten Dokumentenstandards“ ${ }^{*}$.

36 Vgl. aus bibliothekarischer Perspektive und zu den Implikationen für „Bestand“ und „Sammlung“ Kempf 2014, hier: S. 369-371.

37 Vgl. z. B. Neudecker \& Rehm 2016. Eine ausführlichere Einführung am Beispiel der sogenannten Digital Humanities bietet Sabharwal 2015.

38 Obrist \& Raza 2015, S. 38.

39 Bismarck 2004, S. 108. Für uns steht dagegen die klassische Ausstellungskuratierung, wie sie an der SLUB im hauseigenen Buchmuseum mit integrierter „Schatzkammer“ betrieben wird, nicht im Mittelpunkt des Interesses.

40 Stäcker 2019, hier: S. 306.

41 Kempf 2014, S. 385 f.; Ceynowa 2015, hier: S. 269, 270, 274-275.

42 Stäcker 2019, S. 306.

43 Stäcker 2019, S. 307. 
Das Ganze, zu dem diese Tätigkeiten führen sollen, war bisher zumeist auf eine einzelne Institution bezogen. Aktuell zeigt sich, dass der Sammlungsbegriff viel stärker als ein die bibliothekarischen Institutionen ${ }^{44}$ wie auch die anderen Sparten der Gedächtniseinrichtungen ${ }^{45}$ übergreifendes, arbeitsteiliges, teilweise auch kollaboratives Konzept zu denken ist - ein Ansatz, der durchaus einige Bezüge zum Citizen-Science-Gedanken aufweist. Zugleich ändert sich die Gewichtung der damit verbundenen Prozesse: „Sammeln dient nicht mehr vorrangig dem Behalten und Bewahren“ - die ersten beiden Aspekte der Obrist'schen Definition von Kuratieren, die gleichwohl wichtig bleiben -, „sondern vor allem dem erschließenden Aufbereiten, Ordnen und Teilen“46 als Voraussetzung und als Teil der nunmehr zunehmend datengetriebenen wissenschaftlichen Bearbeitung auch im Bereich qualitativer Forschung ${ }^{47}$ als drittem kuratorischen Aspekt nach Obrist. Entsprechend müssen nun auch „die in der Arbeit mit digitalen Inhalten entstehenden Annotationen, Kommentare, Anreicherungen, Veränderungen und Neukontextualisierungen“ ${ }^{48}$ im Kontext des Sammelns als bewusste kuratorische Entscheidung mitbedacht werden. Wer im Rahmen des Konzeptes Open Citizen Science nun diese Arbeit leistet (also welche Akteure), und wo sie geleistet wird (also in welchen Räumen), soll im weiteren Verlauf des Textes näher bestimmt werden. Zunächst gilt es jedoch, die Konzepte Citizen Science und Kuratieren direkt aufeinander zu beziehen.

\subsection{Mit Citizen Science in Wissenschaftlichen Bibliotheken kuratieren}

Mit Bezug auf den Sammlungsbegriff lässt sich das Kuratieren zusammenfassend beschreiben als ein

komplexer zeit- und wissensintensiver Prozess, in dem Experten - z. B. Redakteure, Wissenschaftler oder interdisziplinäre, verteilte Teams - aus einer thematisch typischerweise homogenen, oft aber auch heterogenen Sammlung von Quellen ein neues, in sich kohärentes und abgestimmtes Gesamtwerk entwickeln, das auf einen spezifischen Fokus ausgerichtet ist, also eine spezielle kommunikative Funktion besitzt. ${ }^{49}$

44 Kempf 2014, S. 386-389.

45 Kempf 2014, S. 389-391.

46 Stäcker 2019, S. 309.

47 Koch 2019.

48 Ceynowa 2015, S. 275.

49 Neudecker \& Rehm 2016, S. 104. 
Im Zusammenspiel mit Citizen Science wird deutlich, dass die Beschränkung auf sog. Experten nicht greift bzw. Experten hier nicht als Personen gedeutet werden können, die sich professionell im Sinne von beruflich mit einem Thema auseinandersetzen. Wir reden daher bewusst von kuratorischen Praktiken als einer offeneren Form, die Teilaspekte aus Kuratieren bzw. Kurator herauszieht, ohne das gesamte Bedeutungsfeld mitdenken zu müssen.

Abstrakt formuliert sind kuratorische Entscheidungen in partizipativen Forschungsprojekten von und mit Laien an Bibliotheken Kernelemente der Co-Produktion von neuem Wissen mit Bibliotheksnutzern: Bürgerwissenschaftler/-innen gestalten Forschungsfragen, Recherchen, Wissens- und Objektsammlungen - teils angeleitet und beraten durch Dritte, teils völlig selbstständig. Ein „kohärentes und abgestimmtes Gesamtwerk“ ist dabei nicht in jedem Fall das Ziel der Forschung bzw. von Kuratierung, beispielweise wenn ein Forschungsanliegen im Sinne einer Freizeitbeschäftigung zwar dauerhaft stattfindet, aber einen unbefristeten Charakter hat oder innerhalb eines gleichwohl erkennbar umrissenen Themenfeldes - persönlicher Neugier und persönlichen Interessen folgend - mäandert. Aktivierende Wissenschaftskommunikation kann in beiden Szenarien - Bürgerforschung wie professionelle Forschung - geradezu ansteckend wirken, fachliche Unterstützung ermöglichen oder weitergehende Recherchen anregen. Unwissenschaftlich ausgedrückt: Sie kann Leidenschaften wecken für Forschung und die damit verbundenen Entscheidungen - für und gegen inhärente Optionen: Themen, Forschungsfragen, Quellen, Methoden und Kooperationen.

Die Wirkungen kuratorischer Entscheidungen in Bibliotheken, in privaten Sammlungen und in Citizen-Science-Projekten werden beispielsweise beeinflusst durch:

- Zeit: die Zeitpunkte des Zugangs und der Nutzung von Medien, Daten und anderer Objekte, Zeitverläufe i.S.v. Dauer, gesellschaftlichen Dynamiken, Redaktions-, Publikations- und Embargofristen ${ }^{50}$,

- Methoden und den Stand der Technik in Bezug auf das Sammeln und die Nutzung,

- Konjunkturentwicklungen und Paradigmen der Fachwissenschaften,

50 Dieser Text über Open Citizen Science erscheint in einer Closed-Access-Publikation und kann gemäß den Verlagsrichtlinien erst nach zwölf Monaten frei(er) zugänglich zweitveröffentlicht werden - es sei denn, die Autoren entscheiden sich für einen Freikauf des Artikels, womit er dann im „Hybrid Open Access“ erscheinen kann, in der Regel unter der vergleichsweise restriktiven Lizenz CC BY-NC-ND. Uns ist die Diskrepanz zwischen Titel und Modus der Erstpublikation bewusst. Vgl. die Angaben auf der Verlagshomepage: https://www.degruyter.com/ dg/page/rights-permissions/rechte-lizenzen. Zu den Unterschieden und zur Verwendung der Creative Commons-Lizenzmodelle vgl. Kreutzer 2016. 
- Ressourcen (z. B. Personal, Know-how und Finanzen) der sammelnden und nutzenden Akteure,

- den Grad der Offenheit von Sammlungen, Objekten und ihrer Metadaten technisch wie rechtlich,

- Präsenz und ggf. herausgehobene Präsentation durch Kommunikationsmaßnahmen wie begleitende Social-Media-Aktivitäten ${ }^{51}$,

- Vorlieben unmittelbar und mittelbar beteiligter Akteure und

- auch Zufälle in Sammlungs- und Forschungsprozessen.

Etwas konkreter formuliert gewinnen wir durch das Paradigma der Offenheit in der Bürgerwissenschaft - über vergleichsweise „unprofessionelle Beiträge“ von Bibliotheksnutzer/-innen zum wissenschaftlichen Fortschritt hinaus - potenziell Zugänge bzw. Zugewinne zu den Sammlungen einer Bibliothek:

- Themen- und Nutzungswünsche werden von Nutzern und Nutzerinnen induziert sichtbar.

- Die Sichtbarkeit von Bibliotheks- und Wissensbeständen, von Methoden und Dienstleistungen einer Bibliothek und die Reichweite ihrer Wirkung können wachsen.

- Die Relevanz und der gestiftete Nutzen von gesammelten Beständen, Objekten, Forschungsfragen und Nutzungsszenarien der Metadaten durch Benutzung und Verwendung in neuen Anwendungen und Produkten steigen.

- Wissen entsteht neu durch und mit Dritten auf Basis von institutionell gebundenem Sammlungswissen: dazu gehören das inhärente Wissen von GLAM-Mitarbeiterinnen und -Mitarbeitern und die Metadaten der Bestände zzgl. neuer Verknüpfungen mit anderen Objekten und anderen Sammlungen.

- Nicht zuletzt versprechen sich GLAM-Institutionen Imagegewinne durch intensive Beziehungspflege und Interaktion mit Nutzergruppen.

- Internationale Kooperationen werden wahrscheinlicher, wenn Datenbestände leicht verknüpft werden können.

- Projekt- und Fördermittelakquise können profitieren.

Das bedeutet für Bibliotheken, ihre Nutzer/-innen stärker nicht mehr nur als Konsumenten/Konsumentinnen und Produzenten/Produzentinnen zu verstehen, wenn deren Möglichkeiten und Fähigkeiten wachsen, die verschiedenen Facetten des forschenden Sammelns - durch das Wissenschaftliche Bibliotheken sich auszeichnen - mitzugestalten, vor Ort und räumlich entgrenzt. Sie denken und handeln dann „bibliothekarischer“ als je zuvor und verbinden diese

51 Vgl. zu diesem Aspekt Matzke \& Munke 2020. 
Sicht gleichzeitig mit ihrer Nutzerperspektive als Bürgerwissenschaftler/-innen. Für diesen Wissensaustausch - oder: wechselseitigen Lernprozess - sind spezifische Strategien für die Wissensvermittlung, Beratung der Nutzer/-innen und betriebliche Weiterbildung notwendig. Lernen auf Augenhöhe schließt dann u. U. deutliche Wissensvorteile der Nutzer/-innen gegenüber ihrer Bibliothek bzw. ihrem Personal mit ein. Das ist keine grundsätzlich neue Situation für Forschungsbibliotheken, die schon immer mit und insbesondere für Experten und Expertinnen Fachwissen kuratieren. Professionelle wissenschaftsorientierte Informationsvermittlung muss nun auf größere Nutzergruppen bezogen werden, bei gleichzeitiger Offenheit für wissenschaftliche Methoden und Forschungsergebnisse, die nicht genuin aus dem professionellen Wissenschaftsbetrieb stammen: im Sinne von lebenslangem forschenden Lernen auf beiden Seiten der Wissensbar ${ }^{52}$. Im Falle einer Universitäts- und Landesbibliothek gilt diese Diagnose in beiderlei Hinsicht: landes- und universitätsbibliothekarisch. Die Aufgabenfelder können sich noch stärker aufeinander beziehen, um auch bei der datenorientierten Koproduktion mit den Bürgerwissenschaften voneinander zu profitieren.

\section{Thesen}

Folgende Thesen gründen auf Beobachtungen in der eigenen Bürgerforschung und bibliothekarischen Arbeit der Autoren und ergänzen verallgemeinert eine erste Thesensammlung aus dem Jahr 2018 ${ }^{53}$ :

Der professionellen Wissenschaft fehlt oft das Verständnis für Citizen Science - es sei denn, eigene individuelle Forschungsinteressen sind ggf. betroffen: „Das ist etwas ganz anderes!“ - heißt es dann.

Grenzbereiche zwischen wissenschaftlichem Arbeiten - methodisch begründet und zielgerichtet - und interessengeleiteter, aber dennoch vor allem am eigenen Vergnügen orientierter Recherche sowie eher journalistische Arbeitsweisen mit der Neugier am Geschichtenerzählen - diese Grauzonen sind die Felder bürgerwissenschaftlichen Engagements.

Bibliotheken und ihre Verbünde lieben das Paradigma Führendes System. Dieses Leitbild stößt im Kontext offener Daten in Verbindung mit verteilten Infrastrukturen, Akteuren und partizipativen Methoden an Grenzen. OpenGLAMStrategien erfordern als alternatives Paradigma stattdessen Standards und offe-

52 Unter dem Label Wissensbar betreibt die SLUB ihre Expertenberatung, angelehnt an das Genius Bar-Konzept der Firma Apple. Vgl. Mittelbach \& Muschalek 2015; Mittelbach 2013. 53 Munke \& Bemme 2019a. 
ne dokumentierte Schnittstellen für die Verknüpfung von Sammlungen und Metadaten, um Datenbestände wirksam zu vernetzen. Zugleich kann das für Bibliotheken auch bedeuten, sich zumindest in Teilen vom Gedanken einer „Hoheit“ über die eigenen Daten verabschieden zu müssen.

Vorausgesetzt die Prinzipien und Methoden von Open Science sind die Basis wissenschaftlicher Arbeit, sollten die akademische Beurteilung der wissenschaftlichen Qualität von Forschungsergebnissen und die vergleichende Bewertung von professioneller Forschung und Citizen Science einfacher sein als in anderen, nicht offenen Szenarien. Zugleich bleibt die Frage, ob bürgerwissenschaftlichen Projekten in Bezug auf eigene Handlungsweisen und Rationalitäten ein je eigener Charakter zugestanden werden muss - ohne den Rahmen von Wissenschaftlichkeit zu verlassen? Die Bedürfnisse von und die Erwartungen an forschende Laien unterscheiden sich teilweise von denen der Profis. Darauf können Bibliotheken reagieren, um solche Diskrepanzen zu berücksichtigen.

Denn: Universitäts- und Landesbibliotheken können einen Campus mit der Stadt und der Region vernetzen, in der er sich befindet. Sie wirken dann als Scharnier für Citizen Science, indem sie wissenschaftliche Methoden und bürgerschaftliches Engagement in der Forschung mit dem Paradigma der offenen Wissenschaft verknüpfen und eine Annäherung der verschiedenen Fach- und Forschungskulturen ermöglichen. Bibliotheksmitarbeiter/-innen, die wie Bürgerwissenschaftler/-innen denken und handeln, sind in sehr verschiedenen Rollen unterwegs: kuratierend, als Moderatorinnen/Moderatoren, information professionals, Co-Initiatorinnen und -Initiatoren und Forscher/-innen.

Mehr Investitionen in Ideen und Infrastruktur für forschende Bürger/-innen sind notwendig, um die Wirkung öffentlicher (Retro-)Digitalisierungsprogramme zu maximieren. Nötig wären mehr Bürgerwissen zu digitalen Forschungsmethoden und offenen Werkzeugen und Treffpunkte, an denen dieses Wissen vermittelt wird: analoge und digitale in öffentlichen Bildungs-, Forschungs-, und Kultureinrichtungen sowie im Privaten.

Die Aufgabe von Wissenschaftlichen Bibliotheken liegt dann weniger in Übersetzungsleistungen zwischen forschenden Teilsystemen der Gesellschaft, sondern vielmehr in Vermittlungen zwischen ihnen. Im Idealfall arbeiten professionelle und Laienforscher/-innen mit offenen Daten, Methoden, Werkzeugen und Publikationen, so dass eine Bibliothek und ihre Mitarbeiter/-innen über Informationsvermittlung hinaus bewusst und mit Empathie für beide Ausprägungen und Bedürfnisse wissenschaftlicher Arbeit zwischen Forschenden vermitteln können: Kontakte, gemeinsame Ziele, Sprache (i.S.v. Verständnis für die Fachsprache der anderen) und Kooperationen, z. B. für auf Crowdsourcing aufbauende Forschungsprojekte. 


\section{Digitale Räume für forschende Bürgerinnen und Bürger}

Wie sehen nun entsprechende Angebote von Bibliotheken konkret aus? Ihre digitalen Zentren sind ihre Verzeichnissysteme. Offen lizensierte Sammlungen für retrodigitalisierte Medien ${ }^{54}$ sowie Repositorien für born-digital-Dokumente sind Ressourcen, einerseits. Verknüpft sind die darin enthaltenen Datenobjekte - die nicht nur gedruckte Medien repräsentieren - mittels Metadaten, Signaturen, Barcodes etc. ggf. im dreidimensionalen Gebäudemodell der Bibliothek oder mit einer Bibliothekskatalog-App ${ }^{55}$ als Interface für die mobile Bibliotheksbenutzung. Die SLUB bietet als Landesbibliothek besonders für die auf das Land Sachsen bezogene Forschung digitale Angebote, ${ }^{56}$ in denen kuratorische Praktiken zur Anwendung kommen und die teilweise in Kooperation von Bibliotheksangestellten und Bürgerinnen/Bürgern ausgebaut werden. Andererseits bilden Bibliothekskataloge und darin verzeichnete digitale Bestände mittlerweile nur ein Angebot unter vielen miteinander verbundenen - einige sollen im Folgenden beispielhaft vorgestellt werden. Open Citizen Science ermöglicht hier nicht nur zusätzliche Nutzungen, Anreicherungen und Verknüpfungen. Schließlich besteht für Laien inzwischen - nicht nur theoretisch - die Möglichkeit, selbstständig eigene, offene Nachweissysteme für digitale und nicht digitale Medien und Objekte zu kreieren, zum Beispiel mit Wikidata.

\subsection{Sächsische Bibliografie}

In der Landeskunde bietet die Sächsische Bibliografie Orientierung. Als Regionalbibliografie verzeichnet sie Publikationen nahezu aller Medienarten, die sich inhaltlich auf Sachsen beziehen (Saxonica) ${ }^{57}$. Erarbeitet wird sie in der Hauptsache von Mitarbeiterinnen und Mitarbeitern der SLUB, die Titeldaten erfassen und sie über eine verbale und klassifikatorische Inhaltserschließung mit weiteren Metadaten anreichern. So werden über diese kuratorische Tätigkeit inhaltliche Schwerpunkte wie auch thematische Cluster vor allem der aktuellen, aber

54 In der SLUB kommen aktuell in der Hauptsache die Public Domain Mark und die Creative Commons Lizenz CC BY-SA 4.0 zum Einsatz. Vgl. die Nutzungsbestimmungen für die Digitalen Sammlungen mit Stand vom 1. Oktober 2019, URL: https://nutzungshinweis.slub-dresden.de. 55 URL: https://www.slub-dresden.de/recherche/slub-app/ (09.01.2020).

56 Vgl. einführend Meyer \& Munke 2018.

57 URL: http://swb.bsz-bw.de/DB=2.304/ (09.01.2020). Vgl. Letocha 2006; Letocha 2003/2004, jeweils mit Verweisen auf die ältere Literatur. 
auch der historischen Forschung deutlich: Über die Vorhaben der Massendigitalisierung - in Sachsen v. a. im Rahmen des Landesdigitalisierungsprogramms $(\text { LDP })^{58}$ des Freistaates - werden auch ältere wissenschaftliche Beiträge nachträglich erschlossen und der professionellen wie der ehrenamtlichen Forschung neu zugänglich gemacht.

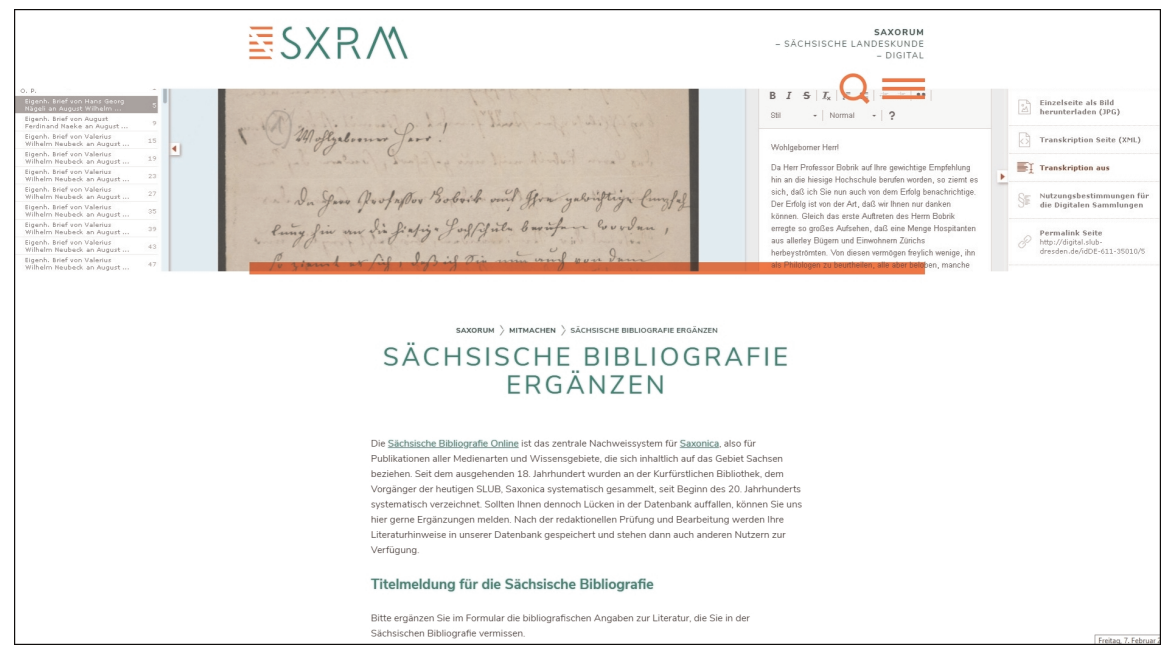

Abb. 2: Titelmeldeformular für die Sächsische Bibliografie im Regionalportal Saxorum: Screenshot. $^{59}$

Ausgehend von der Arbeit an den eigenen Themen können von Nutzerinnen und Nutzern Änderungs- und Korrekturbedarfe an der Regionalbibliografie vorgeschlagen werden ${ }^{60}$ - mittels eines Meldeformulars auf dem Regionalportal Saxorum ${ }^{61}$ oder der Diskussionsseite des Gemeinschaftsaccounts der Bibliografiemitarbeiter/-innen der SLUB in der Wikipedia. ${ }^{62}$ Genutzt werden diese Möglichkeiten von professionellen wie von ehrenamtlichen Forschern: zum Nachweis eigener einschlägiger Publikationen und im Ergebnis der thematischen Literaturrecherche für diese Veröffentlichungen wie für Wikipedia-Artikel. Darüber

58 An der SLUB koordiniert, bezieht das LDP in seiner Programmlinie zur Retrodigitalisierung Kultureinrichtungen aus dem ganzen Freistaat ein. Die Projektergebnisse werden auf dem Portal Sachsen.digital veröffentlicht, https://sachsen.digital. Vgl. Bonte 2016; Meyer \& Munke 2018, hier S. 108-111.

59 https://www.saxorum.de/index.php?id=10207 (31.05.2020).

60 Vgl. Munke 2019a, hier: S. 200-202, 206-207; Munke 2018, hier: S. 309-311.

$61 \mathrm{https}$ //www.saxorum.de (09.01.2020).

62 https://de.wikipedia.org/wiki/Benutzer_Diskussion:SäBi_SLUBDD (09.01.2020). 
hinaus erhalten wir auch Unterstützung bei der Arbeit an Metadaten, wenn etwa Personen wie der Wikipedia-Nutzer Miebner ${ }^{63}$ regelmäßig Hinweise auf im Verbundkatalog falsch verknüpfte Autoren geben oder Informationen zur Anreicherung von Normdatensätzen beisteuern. Für historische Texte, die beispielsweise in Wikisource transkribiert werden, ist es zudem für alle möglich, die Sächsische Bibliografie als Aussage in Wikidata-Items hinzuzufügen und damit durch Wikidata-Abfragen auffindbar und produktiv - im Sinne einer Weiterverarbeitung zu machen. ${ }^{64}$

\subsection{Digitale Sammlungen in Sachsen}

Die SLUB Dresden betreibt für die unterschiedlichen Medienarten - Monografien, Fotos, Grafik, Webseiten - digitale Sammlungen für retro-digitalisierte Medien sowie für born-digital-Publikationen. ${ }^{65}$ Eine Vision lautet, diese - bisher technisch bedingt - unterschiedlichen digitalen Präsentationsräume langfristig zu vereinheitlichen, woran aktuell im Rahmen eines gemeinsam mit der Universitätsbibliothek Leipzig betriebenen, EU-finanzierten Drittmittelprojektes gearbeitet wird. ${ }^{66}$ Durch das bereits genannte Landesdigitalisierungsprogramm LDP wird seit 2015 mit Kultur- und Wissenschaftseinrichtungen ein möglichst umfassender Online-Zugang zu Informationen und Objekten der kulturellen und wissenschaftlichen Überlieferung des Freistaates für Zwecke der Lehre und Forschung sowie für die breite Öffentlichkeit eröffnet. Die Auswahl erfolgt durch Mitarbeiterinnen und Mitarbeiter in den jeweiligen Kultureinrichtungen in Abstimmung mit dem Projektteam der SLUB. Über sporadische Nutzerwünsche wird von Fall zu Fall entschieden. Digitale Werkzeuge für selbstständige Korrekturen digitalisierter Volltexte und für Annotationen stehen bisher in der SLUB noch nicht zur Verfügung. Ihre Entwicklung im Rahmen der für die Digitalisierung und Präsentation verwendeten Softwaresuite Kitodo ${ }^{67}$ ist aber im Kontext des gemeinsam mit der Philipps-Universität Marburg und dem Trier Center for Digital Humanities durchgeführten DFG-finanzierten Drittmittelprojektes zur Di-

63 https://de.wikipedia.org/wiki/Benutzer:Miebner (09.01.2020).

64 Sächsische Bibliografie, Item in Wikidata: (Q61729277).

65 https://digital.slub-dresden.de/, http://www.deutschefotothek.de/, http://mediathek.slubdresden.de, http://www.qucosa.de (09.01.2020).

66 Cross Media Repository. Erweiterung des sächsischen Dokumentenservers Qucosa zu einem Medienübergreifenden Repository, https://slubdd.de/cmr (09.01.2020).

67 https://www.kitodo.org/ (09.01.2020). 
gitalen Edition der Korrespondenz August Wilhelm Schlegels (1767-1845) ${ }^{68}$ vorgesehen.

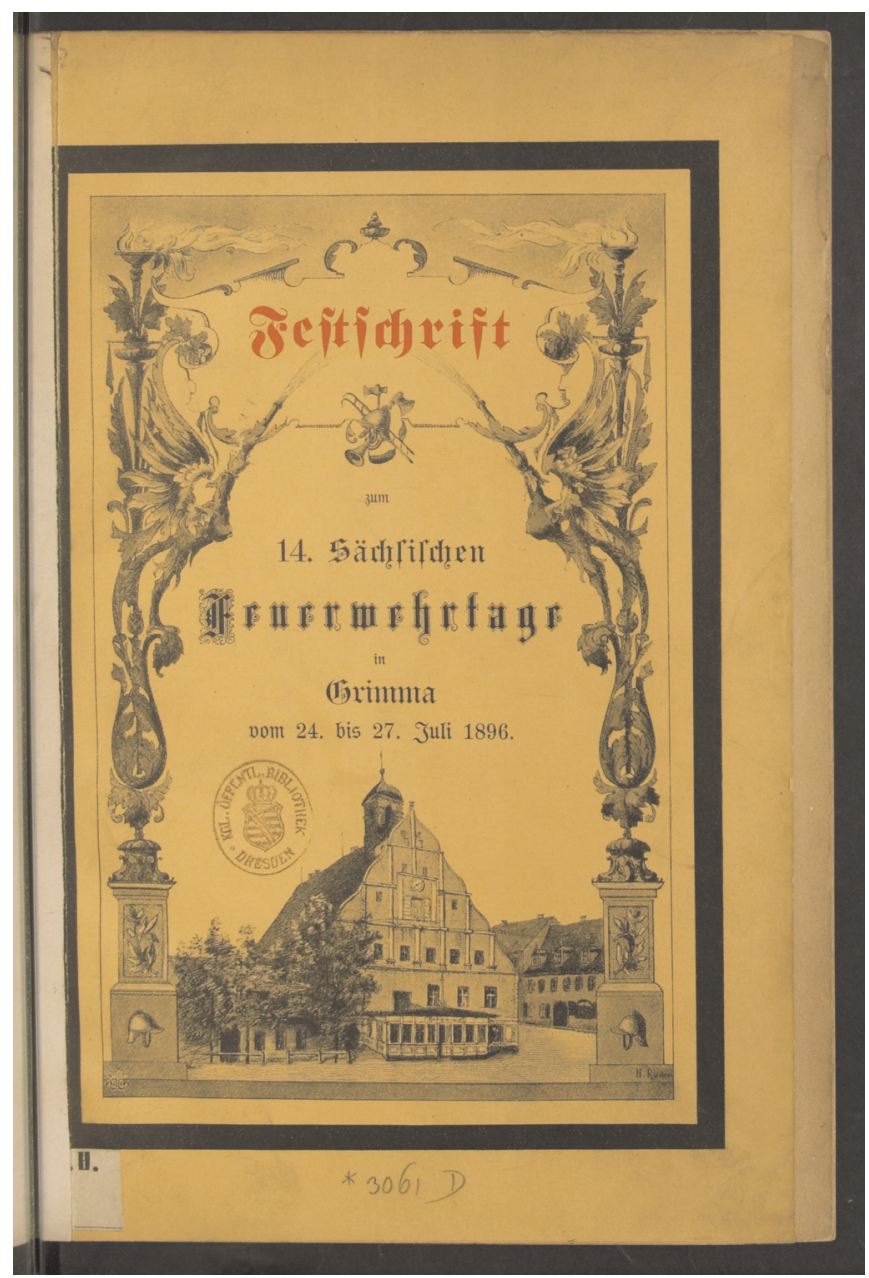

Abb. 3: „Festschrift zum 14. Sächsischen Feuerwehrtage in Grimma vom 24. bis 27. Juli 1896“, Grimma 1896 - ein Beispiel für die Kollektion „Feuerwehrwesen in Sachsen“ der SLUB. ${ }^{69}$

68 https://august-wilhelm-schlegel.de/ (mit Verweisen auf projektbezogene Publikationen) (09.01.2020).

69 https://digital.slub-dresden.de/id399585656/3 (31.05.2020). 
Letztgenanntes Vorhaben richtet sich in der Hauptsache an ein spezialisiertes wissenschaftliches Publikum. Ein anderes Beispiel mit einem eher weiten Publikum zeigt, welche Wirkung diese digitalen Bestände entfalten können: die Kollektion Feuerwehrwesen in Sachsen in den Digitalen Sammlungen der SLUB. ${ }^{70}$ Die Sektion Feuerwehrhistorik ist eine in diesem Zusammenhang relevante Gruppe innerhalb des Sächsischen Landesfeuerwehrverbandes, der 2019 sein 150jähriges Jubiläum feierte. Der Verband verfügt über ein Referat Feuerwehrhistorik und die Berufsfeuerwehr Dresden besitzt eine Abteilung für Traditionspflege. Die Digitalisate der SLUB - deren Auswahl initial durch die Mitarbeiter des Saxonica-Referates erfolgte ${ }^{71}$ - wurden durch diese Gruppen bereits für Publikationen verwendet. Ergänzungen durch Nutzerverweise auf weitere relevante Titel im Bibliotheksbestand, durch Abgabe bisher nicht vorhandener Veröffentlichungen an die Bibliothek sowie durch zusätzliche Forschungsarbeiten konnten im Rahmen der regionalen Fachmesse für Brand- und Katastrophenschutz Florian angeregt werden.

\subsection{SXRM@hypotheses}

Das Weblog Saxorum. Blog für interdisziplinäre Landeskunde in Sachsen ist ein Themenblog im nicht-kommerziellen Blogportal Hypotheses für die Geistes- und Sozialwissenschaften. ${ }^{72}$ Das Portal erleichtert den Betrieb von Wissenschaftsblogs unter einem Dach und sorgt damit für eine größere Sichtbarkeit und Archivierung der Inhalte. Ausgewählte Artikel der verschiedenen Themenportale werden auf der Startseite und in den Social-Media-Kanälen des Portals präsentiert. Saxorum publiziert mit Hypotheses.org Buchrezensionen, Projektberichte und Analysen professioneller Historiker/-innen, Archivarinnen und Archivare, Laien und Autorinnen/Autoren mit landeskundlicher Expertise aus Grenzbereichen der verschiedenen Fach- und Bürgerwissenschaften. Die Ansprache erfolgte zunächst hauptsächlich durch die Redaktion im Referat der Saxonica der SLUB. Mittlerweile erreichen uns auch Themenvorschläge von außen. Artikel von Bürgerwissenschaftlern und -wissenschaftlerinnen (z.B. zu Wikisource-Projekten) und zu Citizen-Science-Themen (z. B. Mobilitätsgeschichte) wurden so bereits publiziert. Die Erschließung der Beiträge erfolgt in der Sächsischen Bibliografie

70 https://digital.slub-dresden.de/kollektionen/1150/ (09.01.2020). Vgl. Hermann \& Munke 2018.

71 Ergänzend zu den Retrodigitalisaten wurde innerhalb der Sächsischen Bibliografie eine Zusammenstellung relevanter aktueller Literatur zur Verfügung gestellt. https://slubdd.de/ feuerwehrbibliografie (09.01.2020).

72 https://saxorum.hypotheses.org/ (09.01.2020). 
und ergänzend in Wikidata in offenen Datenobjekten, die durch jeden bearbeitet werden können.

\subsection{Wikis als kollaborative Gemeinschaften: Wikipedia, Wikisource, Wikidata, Wiki-Commons und Schwesterpro- jekte}

Wikipedia startete im Jahr 2001. ${ }^{73}$ Das Vorläuferportal der heutigen Sprachversionen von Wikisource wurde 2003 gegründet. ${ }^{74}$ Wikidata, die ergänzende multilinguale offene Datenbank, wurde 2019 bereits sieben Jahre alt. Bibliotheken beginnen, die Daten aus Wikidata für ihre Kataloge zu nutzen ${ }^{75}$ - für die Herstellung von Normdatenkonkordanzen, für die Visualisierung raumbezogener Informationen und um mit Bürgerwissenschaftlerinnen und -wissenschaftlern Anwendungen zu entwickeln. Ein Beispiel aus dem Bereich der sächsischen Landeskunde ist die Verknüpfung bisher getrennter Ortsdatenbestände aus dem Historischen Ortsverzeichnis von Sachsen und der Gemeinsamen Normdatei GND via Wikidata. ${ }^{76}$ Nimmt man den gegenwärtigen Stellenwert von Wikipedia für den Erwerb und die Vermittlung von Wissen als Maßstab, ${ }^{77}$ dann steht Wikidata - „die Datenbank, die jeder bearbeiten kann“ - erst am Anfang einer dynamischen Entwicklung. ${ }^{78}$ Wikidata und die zugrundeliegende Softwaresammlung Wikibase $^{79}$ sind grundlegende Technologien, die die Arbeit in Bibliotheken, die Arbeit mit Metadaten und die Kooperation mit Citizen-Science-Projekten bereits verändert hat und zukünftig noch stärker beeinflussen wird. ${ }^{80}$

Offen lizenzierte Daten, beispielsweise von Texten, Dokumenten, Bildern und Klängen zum Thema Sachsen in den Portalen von Wikimedia, werden zunehmend durch strukturierte Datenobjekte in Wikidata beschrieben: WikipediaVerfasser/-innen schaffen enzyklopädische Texte samt ergänzender Medienelemente, Wikisource-Mitarbeiter/-innen transkribieren historische Werke über

73 Vgl. Art. Wikipedia, in: Wikipedia. Die freie Enzyklopädie. https://de.wikipedia.org/wiki/ Wikipedia (09.01.2020).

74 Vgl. Art. Wikisource, in: Wikipedia. Die freie Enzyklopädie. https://de.wikipedia.org/wiki/ Wikisource (15.12.2019).

75 Vgl. z. B. Allison-Cassin \& Scott 2018; Neubert 2017.

76 Munke 2019; Erlinger 2019.

77 Zum Charakter der Wikipedia als Citizen-Science-Projekt vgl. Munke 2018, S. 304-306; Munke 2019a, S. $112 \mathrm{f}$.

78 Bemme 2019.

79 https://wikiba.se/ (09.01.2020).

80 Vgl. Mietchen 2018. 
Sachsen und erschließen diese mit Wikidata formal und inhaltlich, um die Daten mit Wikidata-Abfragen (Queries) auswerten, verarbeiten und visualisieren zu können. Durch skriptbasierte weitgehend automatisierte Verfahren können zusammenhängende Daten gegenüber händischer Bearbeitung zudem in großen Mengen vergleichsweise schnell angereichert werden. Als Saxonica sind diese Informationen und Metadaten grundsätzlich relevant für die Arbeit der SLUB als Landesbibliothek.

Kuratorische Fragen können dabei explizit oder implizit zu entscheiden sein, z. B.:

- Welche Wikisource-Texte werden in welchem Umfang und mit welchen Datenschemata erschlossen?

- Welche Wikidata-Queries werden auf welche Weise abgefragt und visualisiert?

- Wo werden diese Abfragen in anderen Kontexten, wie Webseiten und Anwendungen Dritter, eingebunden oder verarbeitet?

- Welche Themen und Projekte werden in eigenen Social-Media-Kanälen hier: Saxorum, die offiziellen Accounts der SLUB oder in privaten Kommunikationskanälen von Mitarbeitern und Mitarbeiterinnen - hervorgehoben?

- Welche Datenbestände werden auf welche Weise verknüpft?

- Welche Werkzeuge werden für die Anreicherungen genutzt und ggf. weiterentwickelt?

Strategiegeleitete Antworten auf diese Fragen im Kontext von Saxonica sind die Aufgabe einer Landesbibliothek. Sie können sich jedoch zugleich allen stellen, die selbstständig und laienhaft zu landeskundlichen Themen forschen. Beispiel Wikisource: die Jahrgänge 1853 bis 1899 der illustrierten Zeitschrift Die Gartenlaube werden in dem Portal vollständig transkribiert oder auf Basis der OCR korrigiert und mit Wikidata erschlossen. ${ }^{81}$

81 Vgl. Bemme 2019a. 


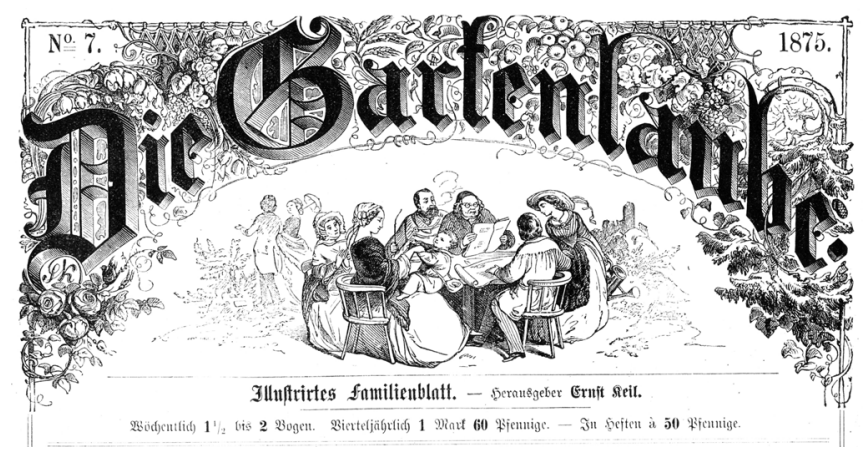

Abb. 4: Ausschnitt des Titelblattes von „Die Gartenlaube“, Nr. 7/1875. ${ }^{82}$

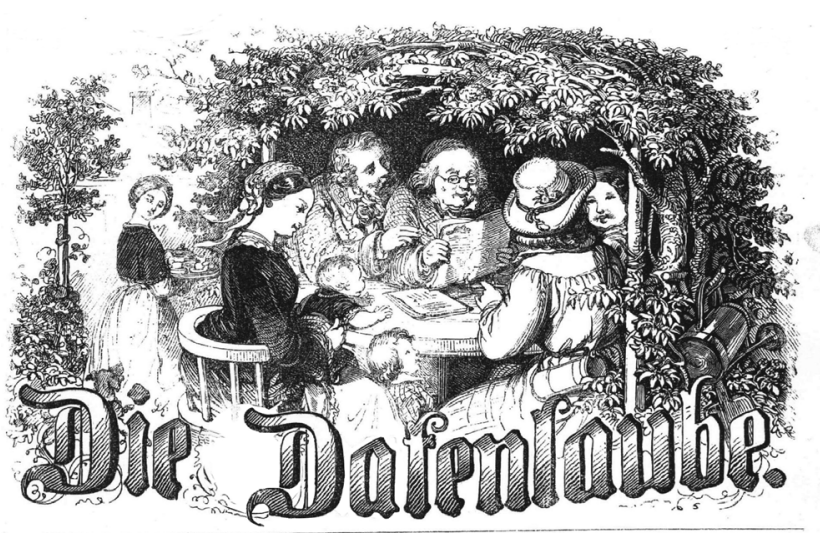

Abb. 5: Logo des Projekts „Die Datenlaube“, basierend auf dem Titelbild von „Die Gartenlaube“, Nr. 1/1853. ${ }^{83}$

Ist dieser Aufwand gerechtfertigt? Finden dabei redundante Arbeiten statt, weil Artikeldaten möglicherweise bereits in der Landesbibliografie enthalten sind? Gemäß etablierter Regeln für die regionalbibliografische Erschließung von Texten und Werken ist die Sächsische Bibliografie gegenüber einer zukünftigen Kompletterschließung der Gartenlaube lückenhaft. Demgegenüber ist es trotzdem naheliegend, die dann ohnehin vorhandenen, durch Bürgerinnen und Bürger erstellten historischen Volltexte, die Sachsen betreffen, regional auch zu verzeichnen und damit besser zugänglich zu machen. Wikidata ist als strukturiertes

82 https://commons.wikimedia.org/wiki/File:Gartenlaube_top.png (31.05.2020). 83 https://commons.wikimedia.org/wiki/File:Die_Datenlaube.xcf. (31.05.2020). 
Datenrückgrat der Wikimediaportale dafür besonders gut geeignet. Ziel ist nun, diese offenen Daten durch definierte Schnittstellen möglichst automatisiert in der traditionellen Sächsischen Bibliografie anzureichern. Der zweite entscheidende Vorteil der Erschließung in Wikidata liegt in den überlegenen Möglichkeiten, solche Daten durch SPARQL-Abfragen ohne großen Aufwand attraktiv zu visualisieren. ${ }^{84}$ Der dritte Vorteil ist die offene Nutzbarkeit von Metadaten der Artikel und Illustrationen aus der Gartenlaube in allen erdenklichen anderen Anwendungen. Ein solcher Anwendungsfall ist die Erstellung von Open Educational Resources (OER) durch Studierende der Universität Leipzig auf Basis von Themen und Texten der Gartenlaube. ${ }^{85}$ Die Rolle der Bibliotheksmitarbeiter ist dabei eine vermittelnde: Idee, informationstechnische Zusammenhänge, Kontakte (z. B. zur Wikisource-Community), Feedback und Öffentlichkeitsarbeit.

\subsection{Stadt-, Regio- und Themen-Wikis}

Drei Stadtwikis gibt es in Sachsen: in Dresden, Freital und Görlitz. ${ }^{86}$ Diese Wikis zeichnen sich insbesondere durch spezifisches Ortswissen aus, das aufgrund der Relevanzkriterien in der Wikipedia ${ }^{87}$ dort möglicherweise nur bedingt oder gar nicht dauerhaft Bestand hätte. Die Auswahl der Themen, die Artikel- und Datenpflege erfolgt ehrenamtlich und ist offen für jeden. Anhand des Stadtwikis Dresden lassen sich Arbeit, Charakter und Selbstbild dieser Bürgerwissenschaftler/-innen neuen Typs i.S. v. Open Citizen Science skizzieren. Diese können mit folgenden Eigenschaften und Themen beispielhaft charakterisiert werden:

- Interesse an Stadt- und Stadtteilgeschichte,

- $\quad$ sammelndes Forschen und Publizieren unter offenen Creative-CommonsLizenzen,

- individuelles, intrinsisch motiviertes Arbeiten an eigenen offenen Wiki-Artikeln in einem gemeinsamen Wiki, das die institutionelle Klammer bildet, jedoch offenbar ohne ein starkes Bedürfnis an Gemeinschaftserlebnisse vor Ort,

84 Vgl. z. B. die Abfrage-basierten Visualisierungen in Erlinger 2019; Munke 2019; Nitzke 2019. 85 Universität Leipzig, Geschichtsdidaktik/HistoDigitaLE im Wintersemester 2019/20, vgl. Wikisource: OER, https://de.wikisource.org/wiki/Wikisource:OER (09.01.2020). Zu den Potenzialen von OER zum Ausbau der didaktischen Rolle von Bibliotheken in der Lehre vgl. Stummeyer 2018.

86 Vgl. Stadtwiki Dresden, Stadtwiki Freital (seit 2015 keine Neuanmeldungen möglich), Stadtwiki Görlitz.

87 Vgl. Art. Relevanzkriterien, in: Wikipedia. Die freie Enzyklopädie. https://de.wikipedia.org/ wiki/Wikipedia:Relevanzkriterien (02.01.2020). 
- IT- und Datenbankaffinität mit Interesse an Wikidata und der Entwicklung von SPARQL-Abfragen.

Das umweltWIKI Sachsen dokumentierte laut Selbstdarstellung das sächsische Umwelt-Engagement seit der friedlichen Revolution im Jahr 1989. Inzwischen ist das Umweltwiki nicht mehr erreichbar, wurde aber wohl teilweise archiviert. ${ }^{88}$

Außerhalb Sachsens ist an Wiki-Vorhaben mit Citizen-Science-Bezug vor allem das GenWiki des Vereins für Computergenealogie zu nennen. ${ }^{89}$ Familienforscher stellen dort einerseits relevante Informationen über ihre Arbeit zur Verfügung, andererseits werden einschlägige Bestände serieller Daten wie Adressbücher, Ortsfamilienbücher oder Familienanzeigen transkribiert. In Kooperation mit dem Verein für Computergenealogie ${ }^{90}$ und dem Dresdner Verein für Genealogie ${ }^{91}$ wird ein gemeinsames Projekt durchgeführt: die Digitalisierung und Erschließung des Dresdner Totengedenkbuchs zum Ersten Weltkrieg, das Informationen zu bis zu 18000 Gefallenen und an Kriegseinwirkungen Gestorbenen enthalten sollte, aber nie publiziert wurde. ${ }^{92}$ Neben den persönlichen Daten wurden u. a. auch Berufswege, Angaben zu militärischen Dienstgraden und zum Einsatz im Ersten Weltkrieg inklusive Auszeichnungen und Verwundungen sowie der Sterbeort, die Todesursache und das Todesdatum abgefragt. Dazu werden die Angehörigen aufgeführt, was den Bestand auch zu einer wichtigen genealogischen Quelle macht - einem zentralen Betätigungsfeld bürgerwissenschaftlichen Forschens. Die Daten sind auf Karteikarten im Sächsischen Staatsarchiv (Hauptstaatsarchiv Dresden - Bestand 11248 Sächsisches Kriegsministerium) und in Angehörigenbriefen im Stadtarchiv Dresden überliefert. Die SLUB hat den Bestand aus dem Hauptstaatsarchiv digitalisiert. Nun werden die Digitalisate in das Datenerfassungssystem des GenWiki übertragen, um anschließend in einem kollaborativen, offenen Projekt transkribiert $\mathrm{zu}$ werden. ${ }^{93}$ Das Vorhaben zeigt zum Abschluss beispielhaft, wie die Arbeit von Bibliotheken in Kontexten von Open Citizen Science auch aussehen kann: Digitalisierung von Beständen anderer Gedächtniseinrichtungen, Erschließung dieser Bestände im System eines bürgerschaftlichen Vereins bei gleichzeitiger Präsentation in den

88 Archive.org: https://web.archive.org/web/20161024110204/http://www.umweltwikisachsen.de/wiki/themen/, nachgewiesen auf https://www.buergerschaffenwissen.de/projekt/ umweltwiki-sachsen (06.10.2019).

89 http://wiki-de.genealogy.net/Hauptseite (09.01.2020).

$90 \mathrm{https} / /$ www.compgen.de/ (09.01.2020).

$91 \mathrm{https} / / /$ www.dresdner-verein-fuer-genealogie.de/ (09.01.2020).

92 Hermann 2014.

93 Vgl. Munke 2019a, S. 115-117, auch mit Verweisen auf ähnliche Projekte z. B. des Sächsischen Staatsarchivs - Staatsarchiv Leipzigs. 
eigenen Digitalen Sammlungen ${ }^{94}$, gemeinsame Netzwerkarbeit im offenen digitalen Raum als Auf- und Ausbau von Kontakten für weitere Vorhaben auch in analogen Räumen.

\section{Offene Räume in Bibliotheken}

Dieser analoge Raum gewinnt für Bibliotheken gerade im Kontext der digitalen Transformation neue Bedeutung. So werden Bibliotheken im Rahmen aktueller Konzepte zur Informationskompetenz ,gerade auch als physischer Ort im Bereich der informellen, offenen und vor allem der selbstgesteuerten Lern- und Arbeits-Settings lokalisiert" ${ }^{\text {95 }}$. Mitglieder des genannten Dresdner Vereins für Genealogie etwa sind in vielfacher Hinsicht in der SLUB präsent: als Ansprechpartner im Rahmen der Wissensbar-Beratung, als Organisatoren und Referenten von öffentlichen Einführungsvorträgen zur Familienforschung, als Aussteller auf dem jährlichen Markt für Dresdner Geschichte und Geschichten ${ }^{96}$ an einem Gemeinschaftsstand mit SLUB-Angestellten und schließlich - neben Wissenschaftlern, Wissenschaftlerinnen und Studierenden - als Beteiligte an internen Workshops zur Ideenfindung für den Relaunch des Internetauftritts der SLUB. Diese Präsenz ist ein Beispiel für den Versuch, den in der SLUB-Strategie verankerten Leitsatz „Menschen machen Bibliotheken. Die SLUB als interaktiver Lern- und Erlebnisraum“ mit Leben zu füllen; auch die Bürgerwissenschaften werden im zugehörigen Abschnitt explizit adressiert. ${ }^{97}$ So kann im Anschluss an Bocklage, Rübenstahl und Siems ,die Bibliothek als Fläche dar[gestellt werden], wo auch andere Akteure Themen bespielen können, die sich mit dem Spektrum der Bibliothek vernetzen lassen und dadurch Mehrwerte generieren" ${ }^{\circ 98}$.

Bibliotheken bieten öffentlichen Raum in durchaus ganz heterogener Ausprägung: sogenannte Dritte Orte ${ }^{99}$ mit Räumen für Begegnungen, Ruhezonen, Raum für individuelle Beratung z. B. in der Wissensbar, Sonderlesesäle, Infotheken, Informationspunkte, Ausstellungsflächen, Carrels, bis zu, nicht unwichtig, Sanitärbereichen. Dieser umbaute Raum definiert Bibliotheken alltagssprach-

94 urn:nbn:de:bsz:14-db-id16628617962 (09.01.2020).

95 Bocklage, Rübenstahl \& Siems 2016, hier: S. 430. Den titelgebenden Begriff des Kuratierens verwenden die Autor/-innen, ohne ihn im Weiteren näher zu bestimmen.

96 Siehe dazu Kap. 5.2 „Dresdner Geschichtsmarkt“.

97 Bonte \& Muschalek 2019, S. 14-17, bes. S. 16.

98 Bocklage, Rübenstahl \& Siems 2016, S. 433.

99 Art. Dritter Ort, in: Wikipedia. Die freie Enzyklopädie: https://de.wikipedia.org/wiki/ Dritter_Ort (09.01.2020). 
lich als physische Orte, auch wenn diese klassische Sicht Änderungen erfährt. Und auch aus der Perspektive von Open Citizen Science bieten umbaute Bibliotheksräume dabei Analogien und Verbindungen für datenbasierte Handlungsfelder.

\subsection{SLUB-Buchmuseum}

Ein Raum innerhalb der SLUB, für den man eine solche Verbindung vielleicht nicht vermuten würde, ist das Buchmuseum. Das Kuratieren von Ausstellungen in klassischer Form, um den vierten Aspekt des Kuratierens nach Obrist zumindest anzureißen, wird in der Bibliothek bereits seit 1935 betrieben. ${ }^{100}$ Nicht nur für die SLUB bzw. die Sächsische Landesbibliothek als ihre Vorgängerinstitution handelt(e) es sich hier um ein traditionelles Thema. Auch für andere Bibliotheken war und ist die Ausstellungstätigkeit ein wichtiges Mittel, um Aufmerksamkeit zu generieren. ${ }^{101}$ Im Kontext der SLUB-Strategie soll nun dieser Raum geöffnet werden, und zwar als „Experimentierraum für Nutzende für mindestens drei Ausstellungsprojekte bis 2025“102. Aktuell geschieht das bereits für die Nutzergruppe der Wissenschaftlerinnen und Wissenschaftler mit einer Ausstellung des Dresdner Sonderforschungsbereiches 1285 Invektivität. ${ }^{103}$ An der Folgeausstellung im Rahmen des sächsischen Jahres der Industriekultur zu den Verbindungen von technischer Bildung ist mit dem Verein für Wissenschaftler und ingenieurtechnische Mitarbeiter Dresden WIMAD e. V. wiederum eine bürgerschaftliche Vereinigung beteiligt. Darüber hinaus bieten die Tätigkeiten in den digitalen Räumen Anknüpfungspunkte - etwa in der Präsentation offener Daten aus Transkriptionsprojekten zur sächsischen Industriekultur wie von Andreas Wagner, der Anreicherung der zugehörigen Datensätze in Wikidata durch interessierte Besucher/-innen oder im interaktiven Einbezug von multimedialen Formaten über Hilfsmittel wie die Magic Box ${ }^{104}$. Jenseits des (bürger)wissenschaftlichen Arbeitens bestand am Rande der Ausstellung „Geteilte Erinnerungen: Das Fotoalbum - Gesteckt, geklebt, gepostet“ die Möglichkeit, sog. Selfies vor durch die Kuratoren bereitgestellten Hintergründen anzufertigen, die Bilder mit einem festgelegten Hashtag in Sozialen Medien zu posten und damit selbst Teil der

$100 \mathrm{Zu}$ Geschichte und Perspektiven vgl. zuletzt Bürger 2009; Nitzschke 2009.

101 Zur Ausstellungspraxis in Bibliotheken vgl. grundlegend Hauke 2016.

102 Bonte \& Muschalek 2019, S. 25.

103 Ellerbrock 2017.

104 Vgl. Stern 2016 und 2018. 
Ausstellung zu werden, da sie im Anschluss als virtuelles Album innerhalb der Ausstellungsräume gezeigt wurden. ${ }^{105}$

\subsection{Dresdner Geschichtsmarkt}

Eine der sichtbarsten Veranstaltungen in Dresden im Bereich Citizen Science ist der jährlich im Winter stattfindende Markt für Dresdner Geschichte und Geschichten als einer „Veranstaltung, bei der ehrenamtliche Historiker die Ergebnisse ihrer Erforschung von Heimatgeschichte vorstellen"106. Seit 2018 wird er an der SLUB durchgeführt, zuvor war er neunmal in der Fakultät Informatik der TU Dresden zu Gast. Beide Veranstaltungsorte sind zunächst durch persönliches Engagement der veranstaltenden Vereinsmitglieder begründet. Für die SLUB ist der Markt darüber hinaus von strategischem Interesse, so dass er 2020 zum dritten Mal als Kooperationsveranstaltung stattfindet und die Bibliothek räumliche wie personelle Ressourcen investiert. In der Hauptsache handelt es sich - neben der Begleitung durch SLUB-Mitarbeiter wie durch Teilnehmer in den Sozialen Medien unter dem Hashtag \#geschichtsmarkt - noch um eine rein analoge Veranstaltung. Nur wenige der gezeigten Poster finden bisher den Weg an die digitale Öffentlichkeit, was auch am Zuschnitt des aktiven Publikums liegen mag: in der Hauptsache ältere Männer. Hier fehlen noch Konzepte, wie die Beratung des SLUB-Teams zu elektronischem Publizieren stärker wirksam werden kann.

\subsection{Makerspace $=$ Citizen Science}

Dass Wissen durch selbstständiges Handeln und Forschen entsteht, greift die SLUB seit 2015 mit dem Motto Wissen kommt von machen! auf. Es steht seitdem für den SLUB-Makerspace ${ }^{107}$ und darüber hinaus für weitere Angebote nicht-textueller Wissensvermittlung, wobei sich die Bibliothek hier „nicht als Dienstleister, sondern als Ort des selbstständigen Arbeitens und der Wissensvermittlung [sieht]“108. Über Formate wie Meetups wird auch ein Publikum außerhalb der

105 Bove 2018.

106 https://www.geschichtsmarkt-dresden.de/ (09.01.2020). Vgl. knapp Munke \& Bemme 2019, S. 190.

107 https://slubdd.de/makerspace (09.01.2020); Vgl. Tiepmar, Mittelbach \& Kaiser 2018; Bonte 2016a, S. 85-94. Bisher bleibt die SLUB die einzige wissenschaftliche Bibliothek in Deutschland, die einen solchen Makerspace - hier in Kooperation mit der Technischen Universität Dresden - betreibt; Späth, Seidl \& Heinzel 2019, hier: S. 40-41.

108 Tiepmar, Dobeleit \& Mittelbach 2019, S. 97. 
Universität angesprochen. Daneben greift das 2018 gegründete SLUB TextLab in seiner Selbstbeschreibung die Formel Makerspace der Worte auf und sieht sich als „offene Werkstatt für sämtliche Arbeiten am Text“109, bezieht den Aspekt des Machens also auch auf textuelle Daten zurück - etwa mit Workshops zu in den Digital Humanities verbreiteten Programmiersprachen wie Python und R oder mit Seminarangeboten zur digitalen Edition über XML-basierte Auszeichnungssprachen wie TEI. Den Ausgangspunkt bildeten die kooperativen Angebote von SLUB und Technischer Universität Dresden zur Schreibberatung, die in der Zweigbibliothek Erziehungswissenschaften der Bibliothek eine gemeinsame räumliche Konkretion fanden. ${ }^{110}$

Solche Makerspaces sind Raumangebote. Citizen Science benennt hier eher die Software-Komponenten F\&E-orientierter Bürgerwissenschaften. Beide Handlungssphären - Campusbibliothek für Forschung mit Texten und Daten einerseits sowie für Entwicklungen mit Materialien und Daten andererseits - lassen sich als Komplementäre verstehen. Wissen entsteht in beiden Handlungsfeldern z.T. unabhängig und außerhalb akademischer Fachkulturen und tradierter Hierarchien selbstständig durch bürgerwissenschaftliche Fragen, Entwürfe, Prototypen und Produkte. Makerspaces in Bibliotheken bieten insbesondere technische Möglichkeiten, von denen Citizen-Science-Projekte profitieren können. Im Umkehrschluss: Methoden der Bürgerwissenschaften bereichern Makerspaces; beide Handlungsfelder können sich ergänzen, bspw. durch Verbindungen zwischen Campus-Communities und Bürgergesellschaft, durch Selbstorganisation, kollaborative Arbeitsweisen und Strategien des Crowdsourcing. Deutlich wird hier beispielhaft: Die Rolle von Bibliotheken und ihre Beiträge zu bürgerwissenschaftlichen Projekten sind vielfältiger geworden.

\section{Leitbild: Open Citizen Science}

\subsection{Herausforderungen}

Eingangs erörterten wir für das Leitbild Open Citizen Science, dass Herausforderungen für Bibliotheken insbesondere darin bestehen, für die Bürgerwissenschaften reale und digitale Räume $\mathrm{zu}$ verknüpfen und diverse Zugänge zu ermöglichen. Offenheit und Kollaboration sind Voraussetzung und zugleich Ziel

109 Vgl. auch das Blog „SLUB TextLab. Schreiben und Digital Humanities“ auf der Plattform Hypotheses, https://textlab.hypotheses.org/ (09.01.2020).

110 Meyer 2018; Santner 2020. 
für Forschungsprozesse in der Bürgerwissenschaft. Idealerweise sollten Bibliotheksmitarbeiter/-innen deshalb zugleich Bürgerwissenschaftler/-innen sein, um Wissen für Citizen Science und für offene Bibliotheks- und digitale Arbeitsumgebungen zu vermitteln.

Offene Bürgerwissenschaft, verstanden als handlungsleitendes Paradigma der Bibliotheksarbeit, bewirkt Veränderungen hinsichtlich der Wahrnehmung und Gestaltung der

- Tätigkeitsfelder und Berufsbilder in Bibliotheken,

- Ausbildungsmöglichkeiten und Zusatzqualifikationen,

- interdisziplinären Zugänge von Einsteigern und Einsteigerinnen,

- institutionellen Vernetzung und der Verbindungen zwischen Wissenschaftsinstitutionen, Bildungsträgern und Vereinen der Bürgergesellschaft,

- administrativen Schnittstellen für die Verknüpfung von kommunalen Aufgaben (z. B. OpenGLAM mit städtischen Kulturinstitutionen) und bürgerwissenschaftsnahem Verwaltungshandeln über amts- und institutionelle Grenzen hinweg

- und damit verknüpften Ressourcenkonflikten.

Grundlegend für offene Bürgerwissenschaft sind Bewusstsein und Sensibilität für Openness und die offene digitale Archivierung von Forschungsergebnissen bzw. Forschungsdaten aus Citizen-Science-Projekten. Wissensverluste durch verlorengegangene Wiki-Projekte schwächen über den Datenverlust hinaus das Vertrauen in kollaborative Werkzeuge. Die Bandbreite der digitalen Fähigkeiten in den Bürgerwissenschaften ist groß, sie sind nicht berechenbar verteilt und reichen von Schülern, Schülerinnen und Studierenden als (meist) Digital Natives bis zu Menschen im hohen Alter, die als Digital Immigrants Zugang suchen zu zeitgenössischen Datenquellen. Diese Zuschreibungen sind nicht universell, d.h. in jeder Gruppe finden sich variantenreiche Ausprägungen von Digital Information Literacy. Für die Bibliotheken kann dies bedeuten, sehr individualisiert vorgehen $\mathrm{zu}$ müssen, um Bürgerwissenschaftler/-innen zu erreichen und zu begleiten.

\subsection{Chancen}

Eine Landesbibliothek kann als Vorbild wirken. Das Leitbild Offene Bürgerwissenschaft dient dabei als role model, für eigene Projekte sowie verstärkend und reflektierend bezüglich offener Methoden und Strategien anderer Akteure in der Landeskunde und darüber hinaus in benachbarten Forschungsgebieten. Wir nutzen Open Citizen Science als programmatischen Ansatz im Referat Saxonica 
für kuratorische Entscheidungen der Bestandsentwicklung, für die Netzwerkarbeit mit Akteuren der Bürgerwissenschaften, in der Zusammenarbeit mit akademischen Kooperationspartnern, für eigene Publikationen und in der Beratung.

Als Leitbild wurde dieses Paradigma nachträglich formuliert, auf Basis längst beobachtbarer Trends im Bibliotheksbereich und eigener Projekterfahrungen. Offene Kulturdaten, deren Nutzung und Pflege bspw. in Wikidata-Anwendungen, sind rückblickend auf diese Weise für uns in der SLUB zu einem neuen landesbibliothekarischen Handlungsfeld geworden, das die Zusammenarbeit mit Bibliotheksnutzern vertieft, die unsere Bibliothek digital bisher z. B. nur von Zuhause aus nutzen. Wissenschaftskommunikation sehen wir als integrales Element dieses Paradigmas. Dazu gehören Webseiten, Social Media, Ausstellungsposter in Open-Access-Repositorien, Vorträge, persönliche Präsenz in relevanten Fachdiskussionen der genutzten Wikimedia-Portale und auf externen Veranstaltungen, eigene Blogposts und Gastbeiträge in Publikationen Dritter, Aufsätze und populärwissenschaftliche Artikel.

Als formuliertes Leitbild vermuten wir über die Landeskunde hinaus, dass andere Kultur- und Gedächtnisinstitutionen sich an Strategien auf Basis von Open Citizen Science orientieren werden. Fokus unserer Arbeit ist es, Open-Science-Methoden in Verbindung mit Saxonica landesweit zu stärken. Als Partner dienen uns dabei Menschen wie Andreas Wagner, deren Enthusiasmus andere anstecken kann:

Durch Zufall hier gelandet, denn meine Freundin hat eine ganze Stange alte Gesetzbücher vor der Vernichtung bewahrt. Diese wurden im Rahmen einer Gemeindereform aussortiert und sollten weggeworfen werden. Nun stehen sie in einem Schrank und sie stellten eine Herausforderung dar, denn das muss ja einen Sinn gehabt haben, diese Bücher zu retten. Vor zwei Jahren begann ich damit, die Bücher einzuscannen, ich dachte mir, vielleicht kann ich die Scans irgendwie verwerten. [...] Vor einiger Zeit dann bin ich durch Zufall auf die Wikisource gestoßen, und die ganze Sache bekam einen Sinn. [...] Jeder kommt an alte Bücher ran, jeder hat einen Rechner zu Hause, mit Prozessoren, irre schnell und mit gigantischen Speichern. Scannt ein wie die Teufel, transkribiert und korrigiert, was das Zeug hält! ${ }^{111}$

Ein Aufruf, dem wir uns als Bibliotheksmitarbeiter wie als (Bürger-)Wissenschaftler gern anschließen.

111 Vgl. Wikisource: Benutzer: A. Wagner, https://de.wikisource.org/w/index.php?title= Benutzer:A._Wagner\&oldid=203709 (05.09.2007). 


\section{Literatur}

Allison-Cassin, Stacy \& Scott, Dan (2018). Wikidata: a platform for your library's linked open data. Code4Lib Journal, 2018(40). https://journal.code4lib.org/articles/13424

Audunson, Ragnar, Hobohm, Hans-Christoph \& Tóth, Maté (2019): ALM in the public sphere: how do archivists, librarians and museum professionals conceive the respective roles of their institutions in the public sphere? Information Research 24(4). http://informationr. net/ir/24-4/colis/colis1917.html

Bartholmei, Stephan (2019). Fünf Jahre zurück, vier Jahre im Blick - Coding da Vinci entwickelt sich. Dialog mit Bibliotheken 31(1), 10-13. urn:nbn:de:101-2019021827

Bartholmei, Stephan (2014). Der erste deutsche Kultur-Hackathon Coding da Vinci. Dialog mit Bibliotheken 26(2), 9-15. urn:nbn:de:101-2015030914

Beagrie, Neil (2006). Digital Curation for Science, Digital Libraries, and Individuals. The International Journal of Digital Curation 1(1), 3-16. DOI:10.2218/ijdc.v1i1.2

Bemme, Jens (2019). ¡Feliz cumpleaños! Wikidata wird 7 - mit Geschenken „made by the community“. SLUBlog, 29. Oktober 2019. https://blog.slub-dresden.de/beitrag/2019/10/29/ feliz-cumpleanos-wikidata-wird-7-mit-geschenken-made-by-the-community/

Bemme, Jens (2019a). Hilfe für die Datenlaube: mit [[Wikisource+Wikidata]] die freie Quellensammlung verbessern. In: Wikimedia Deutschland Blog, 16. Oktober 2019. https://blog. wikimedia.de/2019/10/16/hilfe-fuer-die-datenlaube-mit-wikisourcewikidata-die-freiequellensammlung-verbessern/

Bemme, Jens \& Munke, Martin (2019). Offene Daten und die Zukunft der Bürgerforschung in Wissenschaftlichen Bibliotheken. In: Thomas Bartoschek, Daniel Nüst \& Mario Pesch (Hrsg.), Forum Citizen Science 2019. Die Zukunft der Bürgerforschung (S. 27-39). Münster. DOI: $10.17605 /$ OSF.IO/QHRC4

Bemme, Jens \& Munke, Martin (2019a). Die Zukunft der Bürgerforschung - Rückblick auf das Forum Citizen Science 2019. In: Saxorum. Blog für interdisziplinäre Landeskunde in Sachsen, 11. Oktober 2019. https://saxorum.hypotheses.org/2905

Bismarck, Beatrice von (2004). Curating. In: Christoph Tannert \& Ute Tischler (Hrsg.), Men in Black. Handbuch der kuratorischen Praxis (S. 108.-110). Berlin.

Bocklage, Thorsten, Rübenstahl, Julia \& Siems, Renke (2016). Informationskompetenz als Kuratieren von Wissensräumen. In: Wilfried Sühl-Strohmenger (Hrsg.), Handbuch Informationskompetenz (S. 427-438), 2., überarb. Aufl. Berlin. DOI: 10.1515/9783110403367-041

Bonn, Aletta, Richter, Anett, Vohland Kathrin et al. (2016). Grünbuch Citizen Science Strategie 2020 für Deutschland. Berlin. urn:nbn:de:101:1-20160621985

Bonte, Achim (2016). Aus Sachsen in die Welt - das sächsische Landesdigitalisierungsprogramm. In: Andreas Degkwitz (Hrsg.), Bibliothek der Zukunft - Zukunft der Bibliothek (S. 10-23). Festschrift für Elmar Mittler. Berlin/Boston. DOI:10.1515/9783110464016-003

Bonte, Achim (2016a): Vorstoß in neue Wissensräume. Makerspaces im Leistungsangebot wissenschaftlicher Bibliotheken. In: Konrad Umlauf, Klaus Ulrich Werner \& Andrea Kaufmann (Hrsg.), Strategien für die Bibliothek als Ort (S. 85-94). Festschrift für Petra Hauke. Berlin. DOI:10.1515/9783110481037-006

Bonte, Achim \& Muschalek, Antonie (Hrsg.) (2019). Wissen teilen - Menschen verbinden. SLUB 2025. Strategie der Sächsischen Landesbibliothek - Staats- und Universitätsbibliothek Dresden. Dresden. urn:nbn:de:bsz:14-qucosa2-357501 
Bove, Jens (2018). Ausstellungseröffnung „Geteilte Erinnerungen: Das Fotoalbum - Gesteckt, geklebt, gepostet“. In: SLUBlog, 11. Oktober 2018. https://blog.slub-dresden.de/beitrag/ 2018/10/11/ausstellungseroeffnung-geteilte-erinnerungen-das-fotoalbum-gestecktgeklebt-gepostet/

Bunge, Eva (2019). Wie viel Naturwissenschaft braucht die Bibliothek? Scientific Literacy und Citizen Science in Öffentlichen Bibliotheken. In: Petra Hauke (Hrsg.): Öffentliche Bibliothek 2030. Herausforderungen - Konzepte - Visionen (S. 241-250). Bad Honnef DOI:10.18452/20190

Bunge, Eva (2017). Citizen Science in der Bibliotheksarbeit. Möglichkeiten und Chancen. Wiesbaden.

Bürger, Thomas (2015). Von der Schatzkammer zur digitalen Informationsinfrastruktur. Herausforderungen an die Bibliotheken. In: Katharina Hoins \& Felicitas von Mallinckrodt (Hrsg.), Macht. Wissen. Teilhabe. Sammlungsinstitutionen im 21. Jahrhundert (S. 63-70). Bielefeld. DOI:10.14361/9783839432556-004

Bürger, Thomas (2009). Das Buchmuseum im Google-Zeitalter. Entwicklungen und Möglichkeiten. In: Stephanie Jacobs (Hrsg.), Zeichen - Bücher - Wissensnetze. 125 Jahre Deutsches Buch- und Schriftmuseum der Deutschen Nationalbibliothek (S. 214-220). Göttingen.

Ceynowa, Klaus (2015). Vom Wert des Sammelns und vom Mehrwert des Digitalen. Verstreute Bemerkungen zur gegenwärtigen Lage der Bibliothek. Bibliothek. Forschung und Praxis 39(3), 268-276. DOI:10.1515/bfp-2015-0042

Ellerbrock, Dagmar et al. (2017). Invektivität - Perspektiven eines neuen Forschungsprogramms in den Kultur- und Sozialwissenschaften. Kulturwissenschaftliche Zeitschrift 2(1), 2-24. DOI:10.2478/kwg-2017-0001

Erlinger, Christian (2019). Sächsische Ortsdaten in der Linked Open Data Cloud: Teilautomatisierte Anreicherung und Analyse der HOV-ID in Wikidata, In: Saxorum. Blog für interdisziplinäre Landeskunde in Sachsen, 5. Oktober 2019. https://saxorum.hypotheses.org/2917

Finke, Peter (Hrsg.) (2015). Freie Bürger, freie Forschung. Die Wissenschaft verlässt den Elfenbeinturm, München.

Finke, Peter (2014). Citizen Science. Das unterschätzte Wissen der Laien. Mit einem Nachwort von Ervin Laszlo, München.

Fischer, Barbara (2019). Coding da Vinci oder für eine Renaissance der Allmendekultur mit digitalen Mitteln. In: Lorenz Pöllmann \& Clara Herrmann (Hrsg.), Der digitale Kulturbetrieb. Strategien, Handlungsfelder und Best Practices des digitalen Kulturmanagements (S. 415-430). Wiesbaden. DOI: 10.1007/978-3-658-24030-1_19

Führendes System, in: Wikipedia. Die freie Enzyklopädie. https://de.wikipedia.org/wiki/ Führendes_System

Georgy, Ursula (2019). Möglichkeiten des Crowdsourcings in Bibliotheken durch Digitalisierung. In: Stephan Büttner (Hrsg.), Die digitale Transformation in Institutionen des kulturellen Gedächtnisses. Antworten aus der Informationswissenschaft (S. 95-110). Berlin.

Georgy, Ursula (2018). Open Innovation und Crowdsourcing. Das Management von Offenheit. In: Frauke Schade \& Ursula Georgy (Hrsg.), Praxishandbuch Informationsmarketing: Konvergente Strategien, Methoden und Konzepte (S. 183-198), Berlin/Boston. DOI:10.1515/ 9783110539011-013

Graf, Nicole (2016). Sie wussten mehr! Vielen Dank! „Offenes“ Crowdsourcing im Bildarchiv der ETH-Bibliothek. In: Andreas Bienert \& Benjamin Flesser (Hrsg.), Konferenzband EVA Berlin 2016. Elektronische Medien \& Kunst, Kultur und Historie (S. 163-168). Berlin. 
Hauke, Petra (Hrsg.) (2016). Praxishandbuch Ausstellungen in Bibliotheken, Berlin. DOI:10.1515/9783110475043

Hermann, Konstantin (2014). Das Dresdner Totengedenkbuch 1914-1918. Mitteilungen des Vereins für Sächsische Landesgeschichte e. V. 12(2014), S. 28-45.

Hermann, Konstantin \& Munke, Martin (2018). „Retten - bergen - helfen!“ Historische Quellen zur Geschichte der sächsischen Feuerwehren digital. In: SLUBlog, 10. November 2018. https://blog.slub-dresden.de/beitrag/2018/11/10/retten-bergen-helfen-historischequellen-zur-geschichte-der-saechsischen-feuerwehren-digital/

Ignat, Tiberius, Cavalier, Darlene \& Nickerson, Caroline. (2019). Citizen Science and Libraries: Waltzing towards a collaboration. Mitteilungen der Vereinigung Österreichischer Bibliothekarinnen und Bibliothekare 72(2), 328-336. DOI: 10.31263/voebm.v72i2.304

Kempf, Klaus (2014). Bibliotheken ohne Bestand? Bestandsaufbau unter digitalen Vorzeichen. Bibliothek. Forschung und Praxis 38(3), 365-397. DOI:10.1515/bfp-2014-0057

Kluttig, Thekla (2018). Zur Beteiligung von Archiven am Kultur-Hackathon Coding da Vinci. Ein Bericht und sechs Anmerkungen. Sächsisches Archivblatt. Mitteilungen des Sächsischen Staatsarchivs 2018(2), 23-24.

Koch, Gertraud (2019). Zur Datafication der Wissensproduktion in der qualitativen Forschung. In: Jens Klingner \& Merve Lühr (Hrsg.), Forschungsdesign 4.0. Datengenerierung und Wissenstransfer in interdisziplinärer Perspektive (S. 180-195). Dresden. DOI:10.25366/ 2019.14

Kreutzer, Till (2016). Open Content. Ein Praxisleitfaden zur Nutzung von Creative-CommonsLizenzen, 2. Aufl., Bonn 2016. https://upload.wikimedia.org/wikipedia/commons/c/cd/ Open_Content_-_Ein_Praxisleitfaden_zur_Nutzung_von_Creative-Commons-Lizenzen.pdf Kuwalik, Bernd (2016). Digitales Kuratieren - und dann? In: Andreas Bienert \& Benjamin Flesser (Hrsg.), Konferenzband EVA Berlin (S. 75-82). Elektronische Medien \& Kunst, Kultur und Historie, Berlin.

Letocha, Michael (2006). Vom Versuch einer Litteratur der sächsischen Geschichte zur Sächsischen Bibliographie. Geschichte der landeskundlichen Bibliographien in Sachsen. In: Ludger Syré \& Heidrun Wiesenmüller (Hrsg.), Die Regionalbibliographie im digitalen Zeitalter. Deutschland und seine Nachbarländer (S. 349-366). Frankfurt am Main.

Letocha, Michael (2003). Sächsische Bibliographie im digitalen Zeitalter. Neues Archiv für sächsische Geschichte 74/75 (2003/2004), 455-460.

Lohmeier, Felix \& Jens Mittelbach (2014). Offenheit statt Bündniszwang, Zeitschrift für Bibliothekswesen und Bibliographie 61(4/5), 209-215. DOI:10.3196/1864295014614554

Marcum, Deanna (2014). Archives, Libraries, Museums: Coming Back Together? Information \& Culture. A Journal of History 49(1), 74-89. DOI:10.7560/IC49105

Matzke, Judith \& Munke, Martin (2020). Landesgeschichte und Soziale Medien. Eine Annäherung aus sächsischer Perspektive. In: Hessisches Jahrbuch für Landesgeschichte 70, S. 255-284.

Meyer, Julia (2018). Kafka im Makerspace. Kooperation in der akademischen Schreibberatung. In: Achim Bonte \& Julia Rehnolt (Hrsg.), Kooperative Informationsinfrastrukturen als Chance und Herausforderung. Festschrift für Thomas Bürger zum 65. Geburtstag (S. 289-301), Berlin. DOI:10.1515/9783110587524-032

Meyer, Julia \& Munke, Martin (2018). Digitale Landeskunde für Sachsen. Programme und Projekte an der SLUB Dresden. Bibliotheksdienst 52(2), 106-119. DOI:10.1515/bd-2018-0015 
Mietchen, Daniel (2018). A wiki approach to collecting, curating and managing citizen science data, ScidataCon 2018, Gaborone, Botswana, 5.-8. November 2018. DOI:10.5281/ zenodo. 2560019

Mittelbach, Jens (2013). Eine Wissensbar für die SLUB Dresden. Informationsvermittlung als echte Dienstleistung, BIS 6(3), 180-183. URN: nbn:de:bsz:14-qucosa-130389

Mittelbach, Jens \& Muschalek, Antonie (2015). Wissensbar. Experten beraten passgenau. SLUB Dresden bietet mit der Wissensbar ein personalisiertes Beratungsangebot. In: BuB - Forum Bibliothek und Information 67(6), 374-376.

Munke, Martin (2019). Historische Orte mit offenen Daten: HOV + Wikidata. In: Saxorum. Blog für interdisziplinäre Landeskunde in Sachsen, 16. August 2019. https://saxorum. hypotheses.org/2775

Munke, Martin (2019a). Citizen Science/Bürgerwissenschaften. Projekte, Probleme, Perspektiven am Beispiel Sachsen. In: Jens Klingner \& Merve Lühr (Hrsg.), Forschungsdesign 4.0. Datengenerierung und Wissenstransfer in interdisziplinärer Perspektive (S. 107-124). Dresden. DOI:10.25366/2019.11

Munke, Martin (2019b). Landesbibliographie und Citizen Science. Kooperationsmöglichkeiten für Bibliotheken und Wiki-Communities am Beispiel der Sächsischen Bibliografie. In: Maria Elisabeth Müller, Ulrich Hagenah \& Lars Jendral (Hrsg.), Regionalbibliographien: Forschungsdaten und Quellen des kulturellen Gedächtnisses. Liber amicorum für Ludger Syré (S. 195-207). Hildesheim.

Munke, Martin (2018). Gemeinsam Wissen schaffen. Vernetzte Beiträge von wissenschaftlichen Bibliotheken und Wiki-Communitys für eine digitale Landeskunde. In: Achim Bonte \& Julia Rehnolt (Hrsg.), Kooperative Informationsinfrastrukturen als Chance und Herausforderung. Festschrift für Thomas Bürger zum 65. Geburtstag (S. 302-316). Berlin. DOI:10.1515/9783110587524-033

Munke, Martin \& Bemme, Jens (2019). Bürgerwissenschaften in wissenschaftlichen Bibliotheken. Strategie- und kooperative Projektarbeit, Investitionen in offene Kulturdaten und in Anwenderwissen. o-bib. Das offene Bibliotheksjournal 6(4), 178-203. DOI: 10.5282/o$\mathrm{bib} / 2019 \mathrm{H} 4 \mathrm{~S} 178-203$

Munke, Martin \& Bemme, Jens (2019a). Macht Citizen Science glücklich? Bürgerwissenschaft in wissenschaftlichen Bibliotheken. In: Bürger Künste Wissenschaft. Citizen Science in Kultur und Geisteswissenschaften, 12. März 2019. https://bkw.hypotheses.org/1468 Nassehi, Armin (2016). Digitalisierung der Gesellschaft oder Digitalisierung in der Gesellschaft. In: Medienkorrespondenz, 29. Oktober 2016. https://www.medienkorrespondenz. de/leitartikel/artikel/digitalisierung-der-gesellschaft-oder-digitalisierung-indernbspgesellschaft.html

Neubert, Joachim (2017). Wikidata as a linking hub for knowledge organization systems? Integrating an authority mapping into Wikidata and learning lessons for KOS mappings. In: Philipp Mayr et al. (Hrsg.), Proceedings of the 17th European Networked Knowledge Organization Systems Workshop (NKOS 2017), Thessaloniki, Greece, September 21st, 2017, 14-25. http://ceur-ws.org/Vol-1937/paper2.pdf

Neubert, Joachim (2014). Linked Open Data und die Bibliothekspraxis. Zeitschrift für Bibliothekswesen und Bibliographie 61 (2), 59-67. DOI:10.3196/186429501461217

Neudecker, Clemens \& Rehm, Georg (2016). Digitale Kuratierungstechnologien für Bibliotheken. 027.7. Zeitschrift für Bibliothekskultur 4(2), 104-116. DOI:10.12685/027.7-4-2-158 
Nitzke, Solvejg (2019). Sachsens arboreale Merkwürdigkeiten, oder: Wie man Geschichte(n) verwurzelt. In: Saxorum. Blog für interdisziplinäre Landeskunde in Sachsen, 24. Mai 2019. https://saxorum.hypotheses.org/2396

Nitzschke, Katrin (2009). Bibliotheken als Kunstsammlungen. Das Buchmuseum der SLUB gestern und heute. BIS. Das Magazin der Bibliotheken in Sachsen 2(4), 250-253, URN: nbn:de:bsz:14-qucosa-25828

Nutzungsbestimmungen für die Digitalen Sammlungen mit Stand vom 1. Oktober 2019. https://nutzungshinweis.slub-dresden.de

Obrist, Hans Ulrich \& Raza, Asad (2015). Kuratieren! München.

Pohl, Adrian Pohl \& Danowski, Patrick (2014). Linked Open Data in der Bibliothekswelt - Überblick und Herausforderungen. In: Rolf Griebel, Hildegard Schäffler \& Konstanze Söllner (Hrsg.), Praxishandbuch Bibliotheksmanagement (S. 392-409). Berlin. DOI:10.1515/ 9783110303261.392

Programmierschnittstelle, In: Wikipedia. Die freie Enzyklopädie. https://de.wikipedia.org/ wiki/Programmierschnittstelle

Reiterer, Harald et al. (2016). Blended Library - neue Zugangswege zu den Inhalten wissenschaftlicher und öffentlicher Bibliotheken. Bibliothek. Forschung und Praxis 40 2016(1), 7-20. DOI:10.1515/bfp-2016-0010.

Relevanzkriterien. In: Wikipedia. Die freie Enzyklopädie. https://de.wikipedia.org/wiki/Wikipedia:Relevanzkriterien

Rück, Nicolas (2018). Digitalisierte Geschichte: Zurück zur Quelle mit Wikisource; Teil 1, in: Wikimedia Deutschland Blog, 24. September 2018. https://blog.wikimedia.de/2018/09/ 24/digitalisierte-geschichte-zurueck-zur-quelle-mit-wikisource-teil-1/; Teil 2, in: Ebd., 1. Oktober 2018. https://blog.wikimedia.de/2018/10/01/digitalisierte-geschichte-zurueckzur-quelle-mit-wikisource-teil-2/

Sabharwal, Arjun (2015). Digital Curation in the Digital Humanities. Preserving and Promoting Archival and Special Collections, Waltham, MA.

Sachsen.digital. https://sachsen.digital

Santner, Anita (2020). Das SLUB TextLab: Offene Werkstatt für analoge und digitale Textarbeit. Bibliothek. Forschung und Praxis 44(2), 148-157. DOI:10.1515/bfp-2020-2092

SLUB TextLab (o. J.). Schreiben und Digital Humanities. https://textlab.hypotheses.org/

Späth, Katharina, Seidl, Tobias \& Heinzel, Viktoria (2019). Verbreitung und Ausgestaltung von Makerspaces an Universitäten in Deutschland. In: o-bib. Das Offene Bibliotheksjournal 6 (3), 40-55. DOI:10.5282/o-bib/2019H3S40-55

Stäcker, Thomas (2019). Die Sammlung ist tot, es lebe die Sammlung! Die digitale Sammlung als Paradigma moderner Bibliotheksarbeit. Bibliothek. Forschung und Praxis 43(2), 304310. DOI:10.1515/bfp-2019-2066.

Stadtwiki Dresden. http://www.stadtwikidd.de/

Stadtwiki Freital. http://stadtwiki.fuer-freital.de

Stadtwiki Görlitz. https://www.stadtwiki-goerlitz.de/

Stern, Thomas (2016). It's a kind of magic. Visualisierte Schätze im Foyer der SLUB Dresden. BIS. Das Magazin der Bibliotheken in Sachsen 9(2), 94-95. URN: nbn:de:bsz:14-qucosa276154

Stern, Thomas (2018). MagicBox im Buchmuseum - analog und digital auf einen Blick. In: SLUBlog, 29. September 2018. https://blog.slub-dresden.de/beitrag/2018/09/29/ magicbox-im-buchmuseum-analog-und-digital-auf-einen-blick/ 
Stummeyer, Sabine (2018). OER - Open Educational Resources: Chancen für wissenschaftliche Bibliotheken durch den Einsatz von freien Lehr- und Lernmaterialien in der Hochschullehre. In: Frauke Schade \& Ursula Georgy (Hrsg.), Praxishandbuch Informationsmarketing: Konvergente Strategien, Methoden und Konzepte (S. 303-318). Berlin. DOI:10.1515/ 9783110539011-020

Tiepmar, Jonas, Dobeleit, Daniela \& Mittelbach, Jens (2019). Making als Bestandteil einer Smart Library. Möglichkeiten zur Weiterentwicklung von Bibliotheken. In: Linda Freyberg \& Sabine Wolf (Hrsg.): Smart Libraries - Konzepte, Methoden und Strategien (S. 95-107). Wiesbaden.

Tiepmar, Jonas, Mittelbach, Jens, Kaiser, Melanie et al. (2018). Wissen kommt von Machen. Zukunftsgestaltung in wissenschaftlichen Bibliotheken im Zeitalter der Digitalisierung. Bibliothek - Forschung und Praxis 42(1), 69-82. DOI:10.1515/bfp-2018-0009

Vohland, Katrin \& Göbel, Claudia (2017). Open Science und Citizen Science als symbiotische Beziehung? Eine Gegenüberstellung von Konzepten. TATuP. Zeitschrift für Technikfolgenabschätzung in Theorie und Praxis 26(1/2), 18-24. DOI:10.14512/tatup.26.1-2.18

Wikimedia Deutschland e. V./Open Knowledge Foundation Deutschland e. V. (2019). ABC der Offenheit, Berlin 2019. https://commons.wikimedia.org/wiki/File:ABC_der_Offenheit__Broschüre_(2019).pdf

Alle Internetquellen wurden, wenn nicht anders angegeben, zuletzt am 14.06.2020 aufgerufen. 